\title{
A comparison of numerical methods for solving multibody dynamics problems with frictional contact modeled via differential variational inequalities
}

\author{
Daniel Melanz ${ }^{\mathrm{a}}$, Luning Fang ${ }^{\mathrm{a}}$, Paramsothy Jayakumar ${ }^{\mathrm{b}}$, Dan Negrut ${ }^{\mathrm{a}, *}$ \\ ${ }^{a}$ Department of Mechanical Engineering, University of Wisconsin-Madison \\ 1513 University Avenue, Madison, WI 53706 \\ ${ }^{b}$ US Army RDECOM TARDEC, 6501 E. 11 Mile Road, Warren, MI 48397-5000
}

\begin{abstract}
We investigate two classes of solvers used to determine the time evolution of large systems of rigid bodies that mutually interact through contact with friction. The contact is modeled through a complementarity condition; the friction is posed as a variational problem. The system dynamics is described by a set of differential algebraic equations coupled with differential variational inequalities (DVI). Upon discretization in time, the complementarity conditions enforced at the velocity level are relaxed to obtain a cone complementarity problem (CCP). The solution of the CCP, which becomes the simulation bottleneck, is found by minimizing an equivalent quadratic optimization problem with conic constraints. Herein, we investigate two classes of solvers for this constrained optimization problem. The projected Gauss-Jacobi (PGJ), projected Gauss-Seidel (PGS), and accelerated projected gradient descent (APGD) methods are exponents of the first class of solvers. They are first order, using only costfunction value and gradient information. The second class of solvers is represented by a symmetric cone interior point (SCIP) method and a primal-dual interior point (PDIP) method. These second order methods rely on a Newton step to identify the descent direction and a line search to compute the step size. All five methods draw on parallel computing on Graphics Processing Unit (GPU) cards; the Newton step employs a sparse parallel GPU solver. Two types of numerical experiments, filling and drafting, are carried out to evaluate the performance of the five solution strategies in terms of convergence rate, accuracy, and computational cost. For consistency, all numerical experiments were performed in the same open source code modified to host the five methods of interest.
\end{abstract}

Keywords: Multibody dynamics, Frictional contact problems, Primal-dual interior point method, Parallel computing, Sparse solver, Granular dynamics

\footnotetext{
${ }^{*}$ Corresponding author

Email address: negrut@wisc.edu (Dan Negrut)
} 


\section{Introduction}

This contribution aims at answering the following question: when using a differential variational approach to pose the rigid body frictional contact dynamics problem, are second order optimization methods; i.e., methods that use Hessian information, more expeditious than first order methods; i.e., methods that use only gradient information? This question is relevant in many-body dynamics problems in which the rigid bodies interact through friction and contact forces. The class of many-body systems includes, for instance, granular matter. As pointed out in [1], more than 50\% of the materials processed in industry come in granular form and understanding their dynamics is relevant in a broad spectrum of practical applications such as additive manufacturing, terramechanics, nanoparticle self-assembly, avalanche dynamics, composite materials, pyroclastic flows, formation of asteroids and planets, meteorite cratering; and also industries such as pharmaceuticals, food processing, farming, mining, etc.

Herein, the many-body dynamics problem is posed in a differential variational framework. On the upside, this yields an approach grounded in a sound analytical foundation capable of addressing Painlevé's paradox [2]. On the downside, the numerical solution process calls for solving a large optimization problem that yields at each time step the friction and contact forces active at each contact point. When there are many contacts active in the system, which is the case, for instance, in granular dynamics, the optimization problem becomes a major bottleneck in the numerical solution process. We seek to understand whether using a second order optimization algorithm speeds up the overall numerical solution. Indeed, one can expect that providing more information about the problem at hand; i.e., embedding the Hessian into the solution process, will produce methods that require fewer iterations for solving the aforementioned optimization problem. Yet, second order methods also call for the solution of linear systems, which for many-body dynamics problems are very large. The question is whether the reduction in number of iterations for convergence compensates for the increase in computational effort associated with each iteration of the second order method. It turns out that for relatively smaller problems, the solution based on a second order optimization method, particularly when relying on a fast parallel sparse linear solver for preconditioning, is the better choice. However, as the dimension of the many-body dynamics problem grows, the execution time spent in linear algebra is expected to ultimately eclipse the gains from the reduction in iteration count. These qualitative remarks are backed by a quantitative analysis that requires preliminaries covered in section 2.1 . Therein, we pose the set of differential variational problem whose solution yields the time evolution of many-body systems made up of rigid bodies that interact through friction and contact. The first and second order optimization methods used to solve for the friction and contact forces are presented in Section $\$ 3$. A scaling analysis of these methods is carried out in Section $\$ 4$ in conjunction with a set of benchmark tests known to be challenging from a numerical solution standpoint. Outcomes of a validation study in which we compared numerical results against experimental data are reported in Section 45 . Closing remarks and lessons learned are summarized in Section 86 Finally, the appendix groups derivations that are important from an argument completeness perspective but have secondary relevance in the economy of the study carried out herein. 


\section{Preliminaries, the dynamics of the rigid body in the presence of friction and contact}

\subsection{Posing the equations of motion}

The time-evolution of a collection of $n_{b}$ rigid bodies interacting through friction and contact is described herein using Cartesian coordinates associated with each body $j$, where $1 \leq j \leq n_{b}$. The array of generalized coordinates $\mathbf{q}=\left[\mathbf{r}_{1}^{T}, \varepsilon_{1}^{T}, \ldots, \mathbf{r}_{n_{b}}^{T}, \varepsilon_{n_{b}}^{T}\right]^{T} \in$ $\mathbf{R}^{7 n_{b}}$, and its time derivative $\dot{\mathbf{q}}=\left[\dot{\mathbf{r}}_{1}^{T}, \dot{\varepsilon}_{1}^{T}, \ldots, \dot{\mathbf{r}}_{n_{b}}^{T}, \dot{\varepsilon}_{n_{b}}^{T}\right]^{T} \in \mathbf{R}^{7 n_{b}}$, are used to represent the state of the system, where for body $j, \mathbf{r}_{j}$ and $\varepsilon_{j}$ are the absolute position of the center of mass and the body orientation Euler parameters, respectively. The derivative of the Euler parameters $\dot{\varepsilon}$ can be replaced with a different set of unknowns; i.e., the angular velocity in local coordinates $\bar{\omega}$, formulating the generalized velocity $\mathbf{v}=$ $\left[\dot{\mathbf{r}}_{1}^{T}, \bar{\omega}_{1}^{T}, \ldots, \dot{\mathbf{r}}_{n_{b}}^{T}, \bar{\omega}_{n_{b}}^{T}\right]^{T}$, which can be mapped to $\dot{\mathbf{q}}$ via [3],

$$
\dot{\mathbf{q}}=\mathbf{T}(\mathbf{q}) \mathbf{v} \text {. }
$$

The equation of motion is expressed in matrix form,

$$
\mathbf{M} \dot{\mathbf{v}}=\mathbf{f}(t, \mathbf{q}, \mathbf{v}),
$$

where $\mathbf{M} \in \mathbf{R}^{6 n_{b} \times 6 n_{b}}$ is the generalized mass matrix, and $\mathbf{f}(t, \mathbf{q}, \mathbf{v})$ is the generalized force applied to the system [3, 4].

The presence of bilateral constraints such as spherical joints, revolute joints, translational joints, etc., restricts the relative motion of two rigid bodies. Mathematically, a set of holonomic algebraic equations are induced by the presence of each of these physical joints,

$$
i \in \mathscr{B}: \quad \mathbf{g}_{i}(\mathbf{q}, t)=0
$$

where $\mathscr{B}$ is the set of all bilateral constraints. The presence of the physical joints also induce kinematic constraints at the velocity level obtained by taking a time derivative of the position kinematic constraints of Eq. $3 . \dot{\mathbf{g}}_{i}(\mathbf{q}, t)=\vec{G}_{i}(\mathbf{q}) \mathbf{v}+\partial \mathbf{g}_{i} / \partial t=0$. Enforcing these constraints calls for the presence of a constraint reaction force $\vec{G}_{i}^{T}(\mathbf{q}) \hat{\gamma}_{i, b}$, which is added to the right-hand side of the equations of motion in Eq2 and depends on an unknown Lagrange multiplier $\hat{\gamma}_{i, b}[3,4]$.

For two bodies $A$ and $B$ in contact, $1 \leq A<B \leq n_{b}$, see Figure 1, let $\overline{\mathbf{s}}_{i, A}$ and $\overline{\mathbf{s}}_{i, B}$ be the location of the contact point with respect to the reference frame of body $A$ and $B$, respectively. Since we are concerned with rigid bodies, $A \neq B$. Assuming that the bodies in contact are defined by smooth boundaries, let $\mathbf{n}_{i}$ be the global unit vector denoting the normal direction at the contact points, and $\mathbf{u}_{i}$ and $\mathbf{w}_{i}$ be two unit vectors that span the contact plane at the point of contact. By convention, $\mathbf{n}_{i}$ points towards the interior of $B$, and $\left\{\mathbf{n}_{i}, \mathbf{u}_{i}, \mathbf{w}_{i}\right\}$ form a right-hand reference frame. The contact force, $\mathbf{F}_{i}$, associated with contact $i$ can be decomposed into normal and tangential/frictional components, $\mathbf{F}_{i, N}$ and $\mathbf{F}_{i, T}$, respectively, where $\mathbf{F}_{i, N}=\hat{\gamma}_{i, n} \mathbf{n}_{i}$ and $\mathbf{F}_{i, T}=\hat{\gamma}_{i, u} \mathbf{u}_{i}+\hat{\gamma}_{i, w} \mathbf{w}_{i}$. Here, $\hat{\gamma}_{i, n}, \hat{\gamma}_{i, u}$ and $\hat{\gamma}_{i, w}$ are the magnitude of the force in each direction, and, for as far as body $B$ is concerned, $\hat{\gamma}_{i, n} \geq 0$. That is, if body $B$ comes in contact with any other body, 


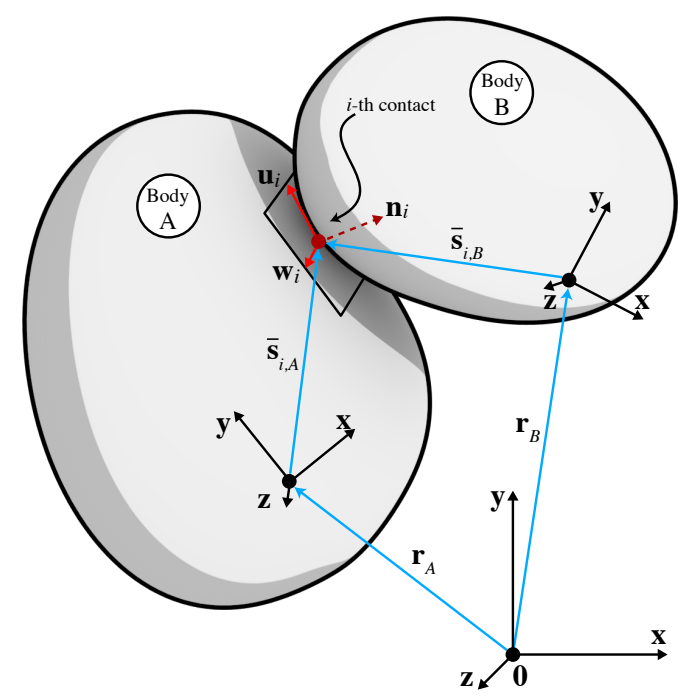

Figure 1: The $i^{\text {th }}$ contact between two bodies $A$ and $B$.

a normal force will act on $B$ to prevent penetration. This defines a unilateral kinematic constraint: body $B$ can move away from body $A$, but it cannot move through it.This unilateral constraint is mathematically posed via the gap function $\phi(\mathbf{q})$ and involves the normal force. Specifically, when two bodies are not in contact, no normal contact force exists; when the bodies are in contact, the contact force is non-negative. This statement is captured in the following complementarity condition,

$$
0 \leq \phi_{i}(\mathbf{q}) \perp \hat{\gamma}_{i, n} \geq 0 \quad \forall i \in \mathscr{A}(\mathbf{q}, \delta),
$$

where $\mathscr{A}(\mathbf{q}, \delta)$ denotes the index set of all pairs of bodies which given a generalized position $\mathbf{q}$ are within a distance less than or equal to $\delta$. We take $\delta>0$ to also include bodies which might come in contact in the immediate future; i.e., during the next time step. The friction force is tied to the value of the normal force via the Coulomb friction model. Using a maximum dissipation principle, see, for instance [5], the Coulomb friction model is posed as the solution of an optimization problem

$$
\left(\hat{\gamma}_{i, u}, \hat{\gamma}_{i, w}\right)=\arg \min \sqrt{\tilde{\gamma}_{i, u}^{2}+\tilde{\gamma}_{i, w}^{2}} \leq \mu_{i} \hat{\gamma}_{i, n} \mathbf{v}_{i, T}^{T}\left(\tilde{\gamma}_{i, u} \mathbf{u}_{i}+\tilde{\gamma}_{i, w} \mathbf{w}_{i}\right),
$$

which provides for contact $i$ the components $\hat{\gamma}_{i, u}$ and $\hat{\gamma}_{i, w}$ of the friction force given a friction coefficient $\mu_{i}$, the relative tangential velocity $\mathbf{v}_{i, T}$ of the two bodies at the contact point, and the normal force at the contact point $\hat{\gamma}_{i, n}$.

A transformation matrix $\mathbf{A}_{i}=\left[\mathbf{n}_{i}, \mathbf{u}_{i}, \mathbf{w}_{i}\right] \in \mathbf{R}^{3 \times 3}$ is used for contact $i$ to express the frictional contact force in the global frame. Additionally, a projection matrix $\mathbf{D}_{i} \in$ $\mathbf{R}^{6 n b \times 3}$,

$$
\mathbf{D}_{i}=\left[\mathbf{0}, \ldots, \mathbf{0},-\mathbf{A}_{i}^{T},-\mathbf{A}_{i}^{T} \mathbf{A}_{A} \tilde{\mathbf{s}}_{i, A}, \mathbf{0}, \ldots, \mathbf{0}, \mathbf{A}_{i}^{T}, \mathbf{A}_{i}^{T} \mathbf{A}_{B} \tilde{\overline{\mathbf{s}}}_{i, B}, \mathbf{0}, \ldots, \mathbf{0}\right]^{T},
$$


is introduced to express the ensuing generalized frictional contact force. Here $\mathbf{A}_{A}$ and $\mathbf{A}_{B}$ are the body $A$ and $B$ rotational matrices, and a tilde over a vector denotes its skewsymmetric matrix [3]. The columns of $\mathbf{D}_{i}$ are denoted by $\mathbf{D}_{i, n}, \mathbf{D}_{i, u}$ and $\mathbf{D}_{i, w}$, therefore, $\mathbf{D}_{i}=\left[\mathbf{D}_{i, n}, \mathbf{D}_{i, u}, \mathbf{D}_{i, w}\right]$.

At this point, a differential variational inequality (DVI) problem can be posed to capture the dynamics of the rigid body in the presence of friction and contact, see for instance [6, 7]:

$$
\begin{aligned}
\dot{\mathbf{q}} & =\mathbf{T}(\mathbf{q}) \mathbf{v} \\
\mathbf{M} \dot{\mathbf{v}} & =\mathbf{f}(t, \mathbf{q}, \mathbf{v})+\sum_{i \in \mathscr{B}} \mathbf{G}_{i}^{T} \hat{\gamma}_{i, b}+\sum_{i \in \mathscr{A}} \mathbf{D}_{i} \hat{\gamma}_{i} \\
i \in \mathscr{B} & : \quad \mathbf{g}_{i}(\mathbf{q}, t)=0 \\
i \in \mathscr{A} & : \quad 0 \leq \phi_{i}(\mathbf{q}) \perp \hat{\gamma}_{i, n} \geq 0 \\
\left(\hat{\gamma}_{i, u}, \hat{\gamma}_{i, w}\right) & =\operatorname{argmin} \sqrt{\tilde{\gamma}_{i, u}^{2}+\tilde{\gamma}_{i, w}^{2} \leq \mu_{i} \hat{\gamma}_{i, n}} \mathbf{v}_{i}^{T}\left(\tilde{\gamma}_{i, u} \mathbf{D}_{i, u}+\tilde{\gamma}_{i, w} \mathbf{D}_{i, w}\right)
\end{aligned}
$$

The right-hand side of the momentum balance equation $(7 \mathrm{~b})$ embeds both the bilateral constraint, $\sum_{i \in \mathscr{B}} \mathbf{G}_{i}^{T} \hat{\gamma}_{i, b}$, and frictional contact forces, $\sum_{i \in \mathscr{A}} \mathbf{D}_{i} \hat{\gamma}_{i}$, where $\hat{\gamma}_{i}=$ $\left[\hat{\gamma}_{i, n}, \hat{\gamma}_{i, u}, \hat{\gamma}_{i, w}\right]^{T}$. The differential attribute of the DVI problem stems from Eqs. $7 \mathrm{a}$ and $7 \mathrm{~b}$. Its variational attribute is associated with the minimization component in Eq. 7e. The inequality aspect goes back to Eq. 7d The problem has also an algebraic attribute that goes back to the set of nonlinear algebraic equations that capture the bilateral constraints. This set of kinematic constraints, which turns an ordinary differential equations problem into an index 3 differential algebraic equations problem, see for instance [8], is important in the economy of the numerical solution yet typically does not get represented in the name DVI associated with the problem in Eq. 7 .

\subsection{The time-stepping scheme}

The numerical solution of the problem stated in Eqs 7 calls for a time discretization. A superscript $(l)$ denotes a variable evaluated at time $t^{(l)}, h$ is the step size, and the contact force impulse is defined as $\gamma_{i,\{n, u, w\}}=h \hat{\gamma}_{i,\{n, u, w\}}$. Following an approach described in [9], the bilateral and unilateral constraints are discretized as follows:

$$
\begin{array}{ll}
i \in \mathscr{B} \quad: \quad \frac{1}{h} \mathbf{g}_{i}\left(\mathbf{q}^{(l)}, t^{(l)}\right)+\mathbf{G}_{i}^{(l)} \mathbf{v}^{(l+1)}+\frac{\partial \mathbf{g}_{i}\left(\mathbf{q}^{(l)}, t^{(l)}\right)}{\partial t}=0 \\
i \in \mathscr{A} \quad: \quad 0 \leq \gamma_{i, n}^{(l+1)} \perp \frac{1}{h} \Phi_{i}\left(\mathbf{q}^{(l)}\right)+\nabla \Phi_{i}\left(\mathbf{q}^{(l)}\right)^{T} \mathbf{v}^{(l+1)} \geq 0 .
\end{array}
$$

Equation 9 relies on a linearization of the unilateral constraint $\Phi_{i}\left(\mathbf{q}^{(l+1)}\right)$. Indeed, given $\mathbf{q}^{(l+1)}=\mathbf{q}^{(l)}+h \mathbf{v}^{(l+1)}, \phi_{i}\left(\mathbf{q}^{(l+1)}\right) \approx \phi_{i}\left(\mathbf{q}^{(l)}\right)+\Phi_{i}\left(\mathbf{q}^{(l)}\right) h \mathbf{v}^{(l+1)} \geq 0$. This leaves the velocity $\mathbf{v}^{(l+1)}$ as the sole variable that needs to adjust at the right of the $\perp$ symbol to meet the complementarity condition. Define next $\gamma_{i}=\left[\gamma_{i, n}, \gamma_{i, u}, \gamma_{i, w}\right]$ as the contact force impulse triplets. As stated in [9], the discretized form of the original set of equations, 
Equations (7a) to (7e) can be formulated as

$$
\begin{aligned}
\mathbf{M}\left(\mathbf{v}^{(l+1)}-\mathbf{v}^{(l)}\right) & =h \mathbf{f}\left(t^{(l)}, \mathbf{q}^{(l)}, \mathbf{v}^{(l)}\right)+\sum_{i \in \mathscr{B}} \mathbf{G}_{i}^{T} \gamma_{i, b}+\sum_{i \in \mathscr{A}} \mathbf{D}_{i} \gamma_{i} \\
i \in \mathscr{B} & : \quad \frac{1}{h} \mathbf{g}_{i}(\mathbf{q}, t)+\mathbf{G}_{i} \mathbf{v}^{(l+1)}+\frac{\partial \mathbf{g}_{i}}{\partial t}=0 \\
i \in \mathscr{A} & : \quad 0 \leq \gamma_{i, n} \perp \frac{1}{h} \phi_{i}\left(\mathbf{q}^{(l)}\right)+\Phi_{i}\left(\mathbf{q}^{(l)}\right) \mathbf{v}^{(l+1)} \geq 0 \\
\left(\gamma_{i, u}, \gamma_{i, w}\right) & =\operatorname{argmin} \sqrt{\bar{\gamma}_{i, u}^{2}+\bar{\gamma}_{i, w}^{2} \leq \mu_{i} \gamma_{i, n}} \mathbf{v}_{i}^{T}\left(\bar{\gamma}_{i, u} \mathbf{D}_{i, u}+\bar{\gamma}_{i, w} \mathbf{D}_{i, w}\right) \\
\mathbf{q}^{(l+1)} & =\mathbf{q}^{(l)}+h \mathbf{T}\left(\mathbf{q}^{(l)}\right) \mathbf{v}^{(l+1)}
\end{aligned}
$$

Equations 10a through 10d combine to form a nonlinear optimization problem with equality constraints. One way to solve this optimization problem is by linearizing the friction cone and turning it into a multifaceted pyramid [6, 10]. Herein, we embrace an alternative method by first introducing a relaxation of the complementarity constraints of Eq. [10c] [9]

$$
0 \leq \gamma_{i, n} \perp \frac{1}{h} \phi_{i}\left(\mathbf{q}^{(l)}\right)+\Phi_{i}\left(\mathbf{q}^{(l)}\right) \mathbf{v}^{(l+1)}-\mu_{i} \sqrt{\left(\mathbf{D}_{i, u}^{T} \mathbf{v}^{(l+1)}\right)^{2}+\left(\mathbf{D}_{i, w}^{T} \mathbf{v}^{(l+1)}\right)^{2}} \geq 0 .
$$

It has been shown in [9] that the solution of the relaxed problem will approach the solution of the original problem as the step size $h \rightarrow 0$. The eventual net effect of the relaxation is that the original highly nonlinear problem can be equivalently posed as a convex problem, more precisely a Cone Complementarity Problem (CCP) of the form [9]

$$
\begin{array}{r}
\text { Find } \gamma_{i}^{(l+1)}, \text { for } i=1, \ldots, N_{c} \\
\text { such that } \Upsilon_{i} \ni \gamma_{i}^{(l+1)} \perp-\left(\mathbf{N} \gamma^{(l+1)}+\mathbf{r}\right)_{i} \in \Upsilon_{i}^{\circ} \\
\text { where } \Upsilon_{i}=\left\{[x, y, z]^{T} \in \mathbb{R}^{3} \mid \sqrt{y^{2}+z^{2}} \leq \mu_{i} x\right\} \\
\text { and } \Upsilon_{i}^{\circ}=\left\{[x, y, z]^{T} \in \mathbb{R}^{3} \mid x \leq-\mu_{i} \sqrt{y^{2}+z^{2}}\right\},
\end{array}
$$

with matrix $\mathbf{N}$ and vector $\mathbf{r}$ defined as

$$
\begin{aligned}
\mathbf{N} & =\mathbf{D}^{T} \mathbf{M}^{-1} \mathbf{D} \\
\mathbf{r} & =\mathbf{b}+\mathbf{D}^{T} \mathbf{M}^{-1} \mathbf{k}
\end{aligned}
$$

where $\mathbf{b}^{T}=\left[\mathbf{b}_{1}^{T}, \ldots, \mathbf{b}_{N_{c}}^{T}\right]$ with $\mathbf{b}_{i}=\left[\phi_{i} / h, 0,0\right]^{T} \in \mathbb{R}^{3}$, and $\mathbf{k}=\mathbf{M} \mathbf{v}^{(l)}+h \mathbf{f}\left(t^{(l)}, \mathbf{q}^{(l)}, \mathbf{v}^{(l)}\right)$.

Solving the CCP of Eq. 12 is equivalent to solving a cone-constrained quadratic optimization (CCQO) problem by considering the KKT first-order necessary conditions, see, for instance, [11]. Given that the problems of interest have tens of bilateral constraints and thousands to millions of unilateral constraints, in what follows we focus on the latter. In this case, the CCQO problem takes the form 


$$
\begin{aligned}
& \min \mathbf{q}(\gamma)=\frac{1}{2} \gamma^{T} \mathbf{N} \gamma+\mathbf{r}^{T} \gamma \\
& \text { subject to } \gamma_{i} \in \Upsilon_{i} \text { for } i=1,2, \ldots, N_{c},
\end{aligned}
$$

where $\gamma_{i}$ is the triplet of multipliers associated with contact $i$; the $\Upsilon_{i}$ is the friction cone of contact $i$; and, $\gamma=\left[\gamma_{1}^{T}, \gamma_{2}^{T}, \ldots, \gamma_{N_{c}}^{T}\right]^{T}$.

The relaxation of the complementarity condition in Eq. 11 has two consequences. First, on the upside, the numerical solution can leverage a wider spectrum of methods to handle the resulting quadratic program with conic constraints in Eq. (15). Second, on the downside, the relaxation embedded in the solution introduces artifacts when the step size $h$ is large and/or the relative sliding velocity between the two bodies in contact is large and/or the friction coefficient at the interface is large. The latter typically is a non-issue since $\mu$ varies in a relatively small range. It should be pointed out that there are approaches that do not fall back on a relaxation, see for instance [12, 13, [14]. A full blown nonlinear complementarity approach is pursued in [13] by means of a Newton method applied in conjunction with an appropriately defined FischerBurmeister complementarity function, an approach that is backed up as a fail-safe by a more robust albeit costlier local solver [15]. A nonsmooth Newton approach is also taken in [12] to solve a fixed point problem.

Ultimately it comes down to either (i) embracing a relaxed approach that leads to a tame numerical problem, e.g., a CCP or linear complementarity problem upon facetization of the friction cone, which both yield a global solution that might displays unphysical artifacts, or (ii) staying faithful to the Coulomb dry friction model and dealing with a numerical problem that in circumstances not known a priori might not have a solution for large and/or complex systems. This paper does not attempt to elucidate this conundrum, a topic discuss extensively in numerous contributions, see, for instance, [16, 17, 18, 19, 20, 21, 22, 23]. Instead, we embrace the relaxation and ensuing CCP approach and focus next on identifying approaches to find the solution of the CCQO problem of Eq. 15 robustly and expeditiously.

\section{Optimization methods considered}

Three first order methods are considered in this study: Projected Jacobi (PJ), Projected Gauss-Seidel (PGS), and Accelerated Projected Gradient Descent (APGD). The first two and variants thereof have been used extensively in the literature [16, 17, 18, 19, 20, 21, 22]. APGD has been introduced recently [24]. PJ, PGS, and APGD will be compared with two recently introduced second-order methods: the Primal-Dual Interior Point Method (PDIP) [25], and the Symmetric Cone Interior Point Method (SCIP) [26].

\subsection{First-order methods: Jacobi, Gauss-Seidel, and Nesterov}

PJ and PGS have been for more than two decades the go-to methods for solving rigid-body dynamics in the presence of friction and contact when embracing a DVI modeling approach [16]. For the relaxed solution approach embraced herein, the 
methodology discussed in [9, 27] solved the CCQO problem of Eq. 15] using the same PJ or PGS algorithms. Between the two, PGS is almost always preferred owing to fast convergence. PJ has been used more often in scenarios where parallel computing is leveraged to speed up the simulation speed. The execution flow for the two algorithms is provided in Appendices A.1 and A.2 Additional implementation details can be found in [28]. GPU implementations of these algorithms are discussed in [19, 29].

Nesterov first proposed accelerated gradient schemes in 1983 [30]. Accelerated gradient descent methods can be seen as simple gradient descent methods with the introduction of 'momentum' in the search direction. Note that in this case, momentum refers to the the concept that the search direction should depend on past iterations in addition to the current iteration. Instead of taking the search direction to be opposite of the gradient direction at the current iteration, the introduction of momentum effectively uses a weighted combination of the current and past gradient directions. The remainder of this section describes the origins of the accelerated projected gradient descent (APGD) method in the gradient descent method, the extension to constrained minimization, the introduction of acceleration, and a series of improvements to solve the targeted problem.

The original gradient descent method, often attributed to Cauchy [31], can be expressed as follows, where $f(\mathbf{x})$ is a smooth function to be minimized and $\alpha_{k}$ is a step size for iteration $k$.

$$
\begin{aligned}
\mathbf{x}_{k+1} & =\mathbf{x}_{k}-\alpha_{k} \nabla f\left(\mathbf{x}_{k}\right) \\
\text { where } \alpha_{k} & =\operatorname{argmin}_{\alpha} f\left(\mathbf{x}_{k}-\alpha \nabla f\left(\mathbf{x}_{k}\right)\right)
\end{aligned}
$$

Under certain conditions, for example if $f(\mathbf{x})$ is convex and Lipschitz continuous, convergence to the global solution can be guaranteed. Note that the scheme in Equations 16 17 solves an unconstrained optimization problem. The gradient descent method can be extended to the projected gradient descent method for solving constrained optimization problems under certain conditions (see, for example, [32]). Projected gradient descent takes the following form, where $\Pi_{\mathscr{C}}$ represents projection onto the convex set $\mathscr{C}$ and $\alpha_{k}$ is the step size which should satisfy some condition to ensure sufficient decrease:

$$
\mathbf{x}_{k+1}=\Pi_{\mathscr{C}}\left(\mathbf{x}_{k}-\alpha_{k} \nabla f\left(\mathbf{x}_{k}\right)\right) .
$$

The projected gradient descent method can be shown to have a sub-linear rate of convergence when the objective function $f(\mathbf{x})$ is convex and $\mathscr{C}$ is a convex set:

$$
f\left(\mathbf{x}_{k}\right)-f\left(\mathbf{x}^{\star}\right) \simeq \mathscr{O}(1 / k)
$$

In [30], Nesterov developed a method with an improved convergence rate of $\mathscr{O}\left(1 / k^{2}\right)$. In fact, the method in [30] was shown to be an 'optimal' first-order method for smooth problems [33] in terms of its performance among all first-order methods, up to a constant. 
The following set of equations represents one iteration of the accelerated gradient descent (AGD) scheme [34]. Note that $\mathbf{y}_{0}=\mathbf{x}_{0} \in \mathbb{R}^{n}, \theta_{0}=1, q \in[0,1]$ is a tuning parameter, and $t_{k}$ is the step size for the current iteration.

$$
\begin{array}{ll}
\mathbf{x}_{k+1} & =\mathbf{y}_{k}-t_{k} \nabla f\left(\mathbf{y}_{k}\right) \\
\theta_{k+1} & \text { solves } \theta_{k+1}^{2}=\left(1-\theta_{k+1}\right) \theta_{k}^{2}+q \theta_{k+1} \\
\beta_{k+1} & =\frac{\theta_{k}\left(1-\theta_{k}\right)}{\theta_{k}^{2}+\theta_{k+1}} \\
\mathbf{y}_{k+1} & =\mathbf{x}_{k+1}+\beta_{k+1}\left(\mathbf{x}_{k+1}-\mathbf{x}_{k}\right)
\end{array}
$$

Assume $f(\mathbf{x})$ is convex and Lipschitz continuous with constant $L$; i.e., $\| \nabla f(\mathbf{x})-$ $\nabla f(\mathbf{y})\left\|_{2} \leq L\right\| \mathbf{x}-\mathbf{y} \|_{2}, \forall x, y \in \mathbb{R}^{n}$. Then, the method described by equations 2023 converges for any $t_{k} \leq 1 / L$. In the above, note that $q=1$ leads to $\theta_{k}=1, \beta_{k}=0$, and $\mathbf{y}_{k}=\mathbf{x}_{k}$ for all $k \geq 0$, which reduces to the gradient descent method. In general, the parameter $q$ can tune the performance of the method depending on the specifics of the objective function $f(\mathbf{x})$. For example, if $f(\mathbf{x})$ is also strongly convex, i.e., $\exists \mu>0: f(\mathbf{x}) \geq f\left(\mathbf{x}^{\star}\right)+(\mu / 2)\left\|\mathbf{x}-\mathbf{x}^{\star}\right\|_{2}^{2}, \forall \mathbf{x} \in \mathbb{R}^{n}$, then the optimal value is $q=\mu / L$, which achieves a linear convergence rate. If the objective function is not strongly convex, or the strong convexity parameter $\mu$ is unknown, then it is often assumed that $q=0$. Note that the original statement of the accelerated method in [30] had $q=0$, so the convergence rate of $\mathscr{O}\left(1 / k^{2}\right)$ is still valid.

The AGD scheme can be extended to constrained optimization in the same way that gradient descent was extended to projected gradient descent. The resulting algorithm, called Accelerated Projected Gradient Descent (APGD) can be expressed by the following set of computations to be performed at each iteration $k \geq 0$. Once again, let $\mathbf{y}_{0}=\mathbf{x}_{0} \in \mathbb{R}^{n}$, and $\theta_{0}=1$.

$$
\begin{aligned}
\mathbf{x}_{k+1} & =\Pi_{\mathscr{C}}\left(\mathbf{y}_{k}-t_{k} \nabla f\left(\mathbf{y}_{k}\right)\right) \\
\theta_{k+1} & \text { solves } \theta_{k+1}^{2}=\left(1-\theta_{k+1}\right) \theta_{k}^{2} \\
\beta_{k+1} & =\frac{\theta_{k}\left(1-\theta_{k}\right)}{\theta_{k}^{2}+\theta_{k+1}} \\
\mathbf{y}_{k+1} & =\mathbf{x}_{k+1}+\beta_{k+1}\left(\mathbf{x}_{k+1}-\mathbf{x}_{k}\right)
\end{aligned}
$$

When $f(\mathbf{x})$ is convex and Lipschitz continuous with constant $L$, then the method described by Equations 2427 converges for any $t_{k} \leq 1 / L$. An equivalent algorithm was proved in [35] to converge with the same $\mathscr{O}\left(1 / k^{2}\right)$ rate as the AGD method. The algorithm has been further improved to utilize adaptive step sizes [36], momentum restart [37], and a fall-back strategy. The complete algorithm can be found in Appendix A.3.

\subsection{Second-order methods}

This section describes the interior point (IP) method for solving the cone-constrained quadratic optimization problem in Equation 15. Recall that the IP method is a second 
order method, and therefore uses information about the second derivative of the objective function, while all of the previously described methods were first order methods, meaning they only used information about the first derivative of the objective function. The remainder of this section will develop the background of IP methods, and then give a PDIP method capable of solving the optimization problem of interest.

In general, IP methods can solve convex optimization problems that include inequality constraints:

$$
\begin{aligned}
& \min f_{0}(\mathbf{x}) \\
& \text { subject to } f_{i}(\mathbf{x}) \leq 0, i=1, \ldots, m
\end{aligned}
$$

In Equation 28, $f_{0}(\mathbf{x}), \ldots, f_{m}(\mathbf{x}): \mathbb{R}^{n} \rightarrow \mathbb{R}$ should be convex and twice continuously differentiable. Then, the Karush-Kuhn-Tucker (KKT) conditions can be expressed as follows, where $\mathbf{x}^{\star} \in \mathbb{R}^{n}$ and $\lambda^{\star} \in \mathbb{R}^{m}$ are the optimal primal and dual respectively.

$$
\begin{aligned}
f_{i}\left(\mathbf{x}^{\star}\right) & \leq 0, \quad i=1, \ldots, m \\
\lambda_{i}^{\star} & \geq 0, \quad i=1, \ldots, m \\
\nabla f_{0}\left(\mathbf{x}^{\star}\right)+\sum_{i=1}^{m} \lambda_{i}^{\star} \nabla f_{i}\left(\mathbf{x}^{\star}\right) & =0 \\
\lambda_{i}^{\star} f_{i}\left(\mathbf{x}^{\star}\right) & =0, \quad i=1, \ldots, m
\end{aligned}
$$

To enable the solution of problem 28 , the constraints are moved into the objective function via an indicator function, resulting in the following equivalent unconstrained problem,

$$
\min f_{0}(\mathbf{x})+\sum_{i=1}^{m} I\left(f_{i}(\mathbf{x})\right),
$$

where $I: \mathbb{R} \rightarrow \mathbb{R}$ is the indicator function for non-positive reals,

$$
I(z)=\left\{\begin{array}{ll}
0 & \text { if } z \leq 0 \\
\infty & \text { if } z>0
\end{array} .\right.
$$

However, the resulting objective function is not differentiable. Therefore, the indicator function is replaced with a differentiable approximation, specifically the logarithmic barrier function

$$
B(z)=-\frac{1}{t} \log (-z),
$$

where $z<0$, and $t>0$ is a parameter which controls the strength of the barrier. Note that $B(z) \rightarrow I(z)$ as $t \rightarrow \infty$. 
In this manner, each of the $m$ constraints can be added to the objective function as $B\left(f_{i}(\mathbf{x})\right)$, resulting in an unconstrained optimization problem which approximates the original problem. Note that the approximation approaches the original problem as $t \rightarrow \infty$.

$$
\min f_{0}(\mathbf{x})+\sum_{i=1}^{m} B\left(f_{i}(\mathbf{x})\right)
$$

\subsubsection{Primal-dual interior point method}

To solve the problem in Equation 36, a Primal-Dual IP method is proposed. This method can be obtained by considering the KKT conditions of problem 36, stated as follows:

$$
\begin{aligned}
f_{i}(\mathbf{x}) & <0, i=1, \ldots, m \\
\nabla f_{0}(\mathbf{x})+\sum_{i=1}^{m}-\frac{1}{t f_{i}(\mathbf{x})} \nabla f_{i}(\mathbf{x}) & =0
\end{aligned}
$$

Now, define $\lambda_{i}=-1 /\left(t f_{i}(\mathbf{x})\right)$, and write the modified KKT conditions as follows:

$$
\begin{aligned}
f_{i}(\mathbf{x}) & <0, i=1, \ldots, m \\
\lambda_{i} & >0, i=1, \ldots, m \\
\nabla f_{0}(\mathbf{x})+\sum_{i=1}^{m} \lambda_{i} \nabla f_{i}(\mathbf{x}) & =0 \\
-\lambda_{i} f_{i}(\mathbf{x}) & =\frac{1}{t}, i=1, \ldots, m
\end{aligned}
$$

Note that the modified KKT conditions in Equations 3942 are very similar to those in Equations 29 32. Only the last equation in each set is different, and it is clear that Equation 42 approaches Equation 32 as $t \rightarrow \infty$.

The PDIP method is obtained by applying Newton's Method to the modified KKT conditions. Here, it is convenient to define the residual of this system of equations for a certain value of $t$ as

$$
\mathbf{r}_{t}(\mathbf{x}, \lambda)=\left[\begin{array}{c}
\nabla f_{0}(\mathbf{x})+\nabla \mathbf{f}(\mathbf{x})^{T} \lambda \\
-\operatorname{diag}(\lambda) \mathbf{f}(\mathbf{x})-\frac{1}{t} \mathbf{1}
\end{array}\right]=\mathbf{0} .
$$

Note that Equation 43 makes use of the following terms:

$$
\mathbf{f}(\mathbf{x})=\left[\begin{array}{c}
f_{1}(\mathbf{x}) \\
\vdots \\
f_{m}(\mathbf{x})
\end{array}\right], \nabla \mathbf{f}(\mathbf{x})=\left[\begin{array}{c}
\nabla f_{1}(\mathbf{x})^{T} \\
\vdots \\
\nabla f_{m}(\mathbf{x})^{T}
\end{array}\right], \mathbf{1}=\left[\begin{array}{c}
1 \\
\vdots \\
1
\end{array}\right]
$$


Newton's method can be applied to solve Equation 43

$$
\left[\begin{array}{l}
\mathbf{x}+\Delta \mathbf{x} \\
\lambda+\Delta \lambda
\end{array}\right]=\left[\begin{array}{l}
\mathbf{x} \\
\lambda
\end{array}\right]-\nabla \mathbf{r}_{t}(\mathbf{x}, \lambda)^{-1} \mathbf{r}_{t}(\mathbf{x}, \lambda)
$$

Rather than computing the Newton step as in Equation 45, it is advantageous to compute the search direction by solving the following linear system:

$$
\left[\begin{array}{cc}
\nabla^{2} f_{0}(\mathbf{x})+\sum_{i=1}^{m} \lambda_{i} \nabla^{2} \mathbf{f}_{i}(\mathbf{x}) & \nabla \mathbf{f}(\mathbf{x})^{T} \\
-\operatorname{diag}(\lambda) \nabla \mathbf{f}(\mathbf{x}) & -\operatorname{diag}(\mathbf{f}(\mathbf{x}))
\end{array}\right]\left[\begin{array}{c}
\Delta \mathbf{x} \\
\Delta \lambda
\end{array}\right]=-\mathbf{r}_{t}(\mathbf{x}, \lambda)
$$

Note that Equation 46 represents one Newton step for the linear system of Equation 43. and a given value of $t>0$. The idea of the Primal-Dual IP method is to drive $t \rightarrow \infty$ as we take Newtons steps of the form in Equation 46. The PD-IP method can be described in pseudocode as follows [38]:

$$
\begin{aligned}
& \text { Algorithm PD-IP }\left(f_{0}, f_{1}, \ldots, f_{m}, \mu \geq 1, \varepsilon\right) \\
& \text { (1) while }\left\|\mathbf{r}_{t}(\mathbf{x}, \lambda)\right\|_{2}>\varepsilon \\
& \text { (2) Compute } t=\frac{\mu m}{\hat{\eta}} \\
& \text { (3) Compute search direction }\left[\Delta \mathbf{x}^{T} \Delta \lambda^{T}\right]^{T} \\
& \text { (4) Compute step length } s>0 \text { via line search } \\
& \text { (5) Update: } \mathbf{x}=\mathbf{x}+s \Delta \mathbf{x}, \lambda=\lambda+s \Delta \lambda \\
& \text { (6) endwhile } \\
& \text { (7) return Solution } \mathbf{x}^{\star}=\mathbf{x}, \lambda^{\star}=\lambda \text {. }
\end{aligned}
$$

In the preceding algorithm, the line search can be performed via backtracking to ensure that $f_{i}(\mathbf{x})<0$ and $\lambda_{i}>0$ are maintained for all $i$. The backtracking will require two parameters, $\alpha, \beta \in(0,1)$.

This is achieved by first directly computing the largest step length, $s_{\max }$, which maintains $\lambda_{i} \geq 0, \forall i$ :

$$
s_{\text {max }}=\min \left\{1, \min \left\{-\lambda_{i} / \Delta \lambda_{i} \mid \Delta \lambda_{i}<0\right\}\right\}
$$

Then, to ensure $\lambda_{i}>0, \forall i$, take $s=0.99 s_{\max }$. Next, repeatedly multiply $s$ by $\beta$ until we achieve $f_{i}(\mathbf{x})<0, \forall i$. Then, continue multiplying $s$ by $\beta$ until the following condition is satisfied:

$$
\left\|\mathbf{r}_{t}(\mathbf{x}+\Delta \mathbf{x}, \lambda+\Delta \lambda)\right\|_{2} \leq(1-\alpha s)\left\|\mathbf{r}_{t}(\mathbf{x}, \lambda)\right\|_{2}
$$

According to [38], it is common to take $\mu=10, \alpha \in[0.01,0.1]$, and $\beta \in[0.3,0.8]$ in the preceding.

Several terms are required when applying the PDIP method to the CCP of Equation 15. Recall that $\gamma=\left[\gamma_{1}^{T}, \gamma_{2}^{T}, \ldots, \gamma_{N_{c}}^{T}\right]^{T}$, where $\gamma_{i}=\left[\gamma_{i, n}, \gamma_{i, u}, \gamma_{i, v}\right]^{T}$.

First, the objective function in this case is

$$
f_{0}(\gamma)=\frac{1}{2} \gamma^{T} \mathbf{N} \gamma+\mathbf{r}^{T} \gamma
$$


Define $\mathbf{f}(\gamma)$ as follows:

$$
f_{i}(\gamma)= \begin{cases}\frac{1}{2}\left(\gamma_{i, u}^{2}+\gamma_{i, v}^{2}-\mu_{i}^{2} \gamma_{i, n}^{2}\right) & : i \in\left[1, \ldots, N_{c}\right] \\ -\gamma_{\left(i-N_{c}\right), n} & : i \in\left[N_{c}+1, \ldots, 2 N_{c}\right]\end{cases}
$$

Therefore,

$$
\nabla f_{i}(\gamma)=\left[\begin{array}{c}
0 \\
\vdots \\
0 \\
-\mu_{i}^{2} \gamma_{i, n} \\
\gamma_{i, u} \\
\gamma_{i, v} \\
0 \\
\vdots \\
0
\end{array}\right], i=1, \ldots, N_{c}, \text { and } \nabla f_{i}(\gamma)=\left[\begin{array}{c}
0 \\
\vdots \\
0 \\
-1 \\
0 \\
0 \\
0 \\
\vdots \\
0
\end{array}\right], i=N_{c}+1, \ldots, 2 N_{c} .
$$

The following expressions for the second derivatives can be obtained.

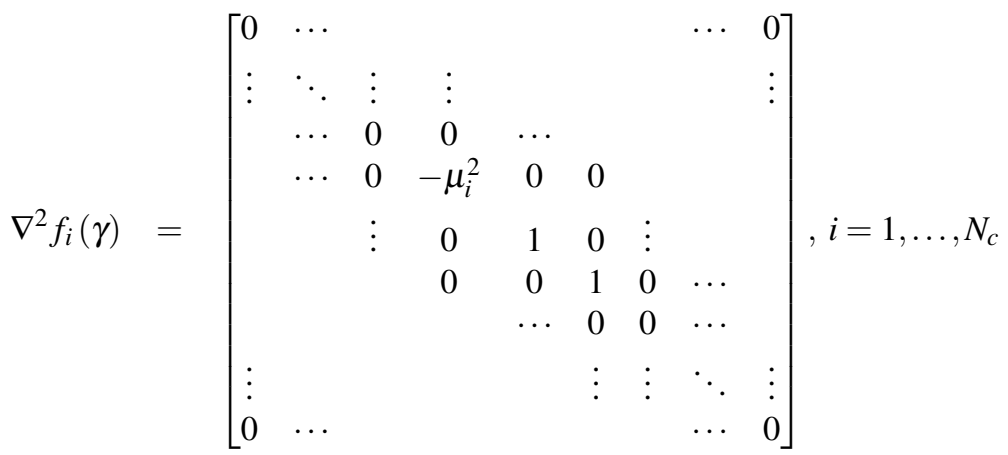

$$
\begin{aligned}
& \nabla^{2} f_{i}(\gamma)=\mathbf{0}_{\left(3 N_{c} \times 3 N_{c}\right)}, i=N_{c}+1, \ldots, 2 N_{c}
\end{aligned}
$$

Let $\mathbf{A}$ be the Newton step matrix as in Equation 46 Then we have the following, where $\mathbf{B}=\nabla \mathbf{f}(\gamma)^{T} \in \mathbb{R}^{3 N_{c} \times 2 N_{c}}, \mathbf{C}=-\operatorname{diag}(\lambda) \nabla \mathbf{f}(\gamma) \in \mathbb{R}^{2 N_{c} \times 3 N_{c}}$, and $\mathbf{D}=-\operatorname{diag}(\mathbf{f}(\gamma)) \in$ $\mathbb{R}^{2 N_{c} \times 2 N_{c}}$ :

$$
\mathbf{A}=\left[\begin{array}{cc}
\mathbf{N}+\hat{\mathbf{M}} & \mathbf{B} \\
\mathbf{C} & \mathbf{D}
\end{array}\right]
$$

Note that the matrix $\hat{\mathbf{M}}$ is defined as follows:

$$
\begin{aligned}
& \hat{\mathbf{M}}=\sum_{i=1}^{2 N_{c}} \lambda_{i} \nabla^{2} \mathbf{f}(\gamma)=\operatorname{diag}(\hat{\mathbf{m}}) \in \mathbb{R}^{3 N_{c} \times 3 N_{c}} \\
& \hat{\mathbf{m}}=\left[-\mu_{1}^{2} \lambda_{1}, \lambda_{1}, \lambda_{1},-\mu_{2}^{2} \lambda_{2}, \lambda_{2}, \lambda_{2}, \ldots,-\mu_{N_{c}}^{2} \lambda_{N_{c}}, \lambda_{N_{c}}, \lambda_{N_{c}}\right]^{T} \in \mathbb{R}^{3 N_{c}}
\end{aligned}
$$


Next, let the right hand side of the Newton step matrix $\mathbf{r}_{t}$ (see Equation 46) be partitioned as follows:

$$
\mathbf{r}_{t}=\left[\begin{array}{l}
\mathbf{r}_{d} \\
\mathbf{r}_{g}
\end{array}\right]
$$

Then, the Newton step for this problem is expressed as follows:

$$
\mathbf{A}\left[\begin{array}{c}
\Delta \gamma \\
\Delta \lambda
\end{array}\right]=-\mathbf{r}_{t}
$$

\subsubsection{Symmetric cone interior point method}

The SCIP is formulated similar to the PDIP algorithm in Section 3.2.1. Firstly, the $\mathrm{CCP}$ is interpreted as a minimization of a potential function. The potential function can be split into two parts, one part being a barrier function that penalizes points close to the boundary of a feasible region. The points furthest away from the boundary in a certain sense are called the central path. As a second step, the idea behind the central path and path-following is presented. Thirdly, a way of constructing a feasible interior starting point for the method is explained. As a fourth step, the maximum step length of the algorithm is discussed. Finally, Nesterov-Todd scaling is introduced to improve the convergence rate.

This method makes use of the algebraic structure presented in [26], specifically with the space $A=\mathbb{R}^{3}$ together with the Jordan product

$$
\mathbf{x} \circ \mathbf{y}=\frac{1}{\sqrt{2}}\left[\begin{array}{c}
\mathbf{x}^{T} \mathbf{y} \\
x_{\mathbf{n}} \mathbf{y}_{\mathbf{t}}+y_{\mathbf{n}} \mathbf{x}_{\mathbf{t}}
\end{array}\right] \in \mathbb{R} \times \mathbb{R}^{2}
$$

for all

$$
\mathbf{x}=\left[\begin{array}{c}
x_{\mathbf{n}} \\
\mathbf{x}_{\mathbf{t}}
\end{array}\right], \mathbf{y}=\left[\begin{array}{c}
y_{\mathbf{n}} \\
\mathbf{y}_{\mathbf{t}}
\end{array}\right] \in \mathbb{R} \times \mathbb{R}^{2}
$$

and relies on the fact that the cone is symmetric, $\Upsilon_{i}=\Upsilon_{i}^{\circ}$ :

$$
\Upsilon_{i}:=\{\mathbf{x} \circ \mathbf{x} \mid \mathbf{x} \in A\}=\left\{\left[\begin{array}{l}
x_{\mathbf{n}} \\
\mathbf{x}_{\mathbf{t}}
\end{array}\right] \in \mathbb{R} \times \mathbb{R}^{2} \mid x_{\mathbf{n}} \geq\left\|\mathbf{x}_{\mathbf{t}}\right\|\right\}
$$

Of course, unless $\mu_{i}=1 \forall i=1, \ldots, n$, the cones $\Upsilon_{i}$ and $\Upsilon_{i}^{\circ}$ must first be transformed using

$$
\mathbf{x}=T_{x} \cdot \gamma=\left[\begin{array}{ccc}
T_{\mu_{1}}^{x} & & \\
& \ddots & \\
& & T_{\mu_{n}}^{x}
\end{array}\right] \cdot \gamma, \mathbf{y}=T_{y} \cdot \mathbf{u}=\left[\begin{array}{lll}
T_{\mu_{1}}^{y} & & \\
& \ddots & \\
& & T_{\mu_{n}}^{y}
\end{array}\right] \cdot \mathbf{u}
$$

with

$$
T_{\mu_{i}}^{x}=\left[\begin{array}{lll}
\mu_{i} & & \\
& 1 & \\
& & 1
\end{array}\right], T_{\mu_{i}}^{y}=\left[\begin{array}{lll}
1 & & \\
& \mu_{i} & \\
& & \mu_{i}
\end{array}\right]
$$


and $\bar{N}=T_{y} N T_{x}^{-1}$ and $\overline{\mathbf{r}}=T_{y} \mathbf{r} \in \mathbb{R}^{3 n}$, so that the CCP can be rewritten as

$$
\Upsilon \ni \mathbf{x} \perp \mathbf{y}=\bar{N} \mathbf{x}+\overline{\mathbf{r}} \in \Upsilon .
$$

As in [26], it is convenient for the definition of the central path to minimize a potential function with a logarithmic barrier for the set

$$
\Upsilon \cup(-\Upsilon)=\left\{\mathbf{x}=\left[\begin{array}{c}
\mathbf{x}_{1} \\
\vdots \\
\mathbf{x}_{n}
\end{array}\right] \in \mathbb{R}^{3 n} \mid \operatorname{det}\left(\mathbf{x}_{i}\right) \geq 0 \forall i=1, \ldots, n\right\},
$$

where

$$
\operatorname{det}(\mathbf{x}):=\frac{1}{2}\left(x_{\mathbf{n}}^{2}-\left\|\mathbf{x}_{\mathbf{t}}\right\|^{2}\right)
$$

rather than using a logarithmic barrier for $\Upsilon$ as in Equation 36. This gives rise to the minimization of the potential function

$$
f(\mathbf{x}, \mathbf{y})=(2 n+\rho) \log \mathbf{x}^{T} \mathbf{y}-2 n \log n-\sum_{i=1}^{n} \log \left(2 \operatorname{det}\left(\mathbf{x}_{i}\right)\right)-\sum_{i=1}^{n} \log \left(2 \operatorname{det}\left(\mathbf{y}_{i}\right)\right),
$$

where $\rho>0$ is an arbitrary constant. The first two terms are a scaled version of the cost function. The last two terms act as a logarithmic potential that drives $\mathbf{x}_{i}$ and $\mathbf{y}_{i}$ away from the manifold defined by $\operatorname{det}\left(\mathbf{x}_{i}\right)=0, \operatorname{det}\left(\mathbf{y}_{i}\right)=0, i=1, \ldots, n$, i.e. the boundary of the double cone $\Upsilon \cup(-\Upsilon)$. We split the potential function into two parts via

$$
\begin{gathered}
f(\mathbf{x}, \mathbf{y})=\rho \log \mathbf{x}^{T} \mathbf{y}+f_{\text {cen }}(\mathbf{x}, \mathbf{y}), \\
f_{c e n}(\mathbf{x}, \mathbf{y})=2 n \log \frac{\mathbf{x}^{T} \mathbf{y} / n}{\prod_{i=1}^{n}\left[2 \sqrt{\operatorname{det}\left(\mathbf{x}_{i}\right) \operatorname{det}\left(\mathbf{y}_{i}\right)}\right]^{1 / n}} .
\end{gathered}
$$

The logarithmic barrier $f_{c e n}$ penalizes values close to the boundary since the denominator in the logarithm in Equation 69 tends towards zero as $(\mathbf{x}, \mathbf{y})$ approaches the boundary of the feasible set. Let $\left(\mathbf{x}^{(k)}, \mathbf{y}^{(k)}\right)$ be a sequence in the feasible set that approaches an optimum $\left(\mathbf{x}^{*}, \mathbf{y}^{*}\right)$ of Equation 15 . If $f_{c e n}\left(\mathbf{x}^{(k)}, \mathbf{y}^{(k)}\right)=0$, the sequence approaches the boundary of the feasible set from the interior as fast as it decreases the cost function and therefore the sequence approaches the optimum strictly from within the feasible set, staying clear from the constraints. If $f_{c e n}\left(\mathbf{x}^{(k)}, \mathbf{y}^{(k)}\right)=0$, the constraints are eliminated from the potential function. It is reduced to a scaled logarithm of the cost function. The central path is defined as

$$
\begin{aligned}
S_{c e n}:= & \left\{(\mathbf{x}, \mathbf{y}) \in \Upsilon \cup(-\Upsilon) \mid f_{\text {cen }}(\mathbf{x}, \mathbf{y})=0\right\} \\
& =\{(\mathbf{x}, \mathbf{y}) \in \Upsilon \cup(-\Upsilon) \mid \mathbf{x} \circ \mathbf{y}=\alpha \mathbf{e}\}
\end{aligned}
$$

In practice it is not possible to rigorously enforce $f_{c e n}\left(\mathbf{x}^{(k)}, \mathbf{y}^{(k)}\right)=0$. Instead, one tries to find a sequence in a small neighborhood of the central path. A Newton step applied to the function

$$
\mathbf{g}(\mathbf{x}, \mathbf{y})=\mathbf{x} \circ \mathbf{y}-\alpha \mathbf{e}=\mathbf{0}
$$


is a step towards the central path at $\alpha$. Given a feasible $\left(\mathbf{x}^{(k)}, \mathbf{y}^{(k)}\right)$, the search direction of the method is given by a solution to

$$
\left[\begin{array}{cc}
\nabla_{\mathbf{x}} \mathbf{g}\left(\mathbf{x}^{(k)}, \mathbf{y}^{(k)}\right) & \nabla_{\mathbf{y}} \mathbf{g}\left(\mathbf{x}^{(k)}, \mathbf{y}^{(k)}\right) \\
\bar{N} & -I
\end{array}\right]\left[\begin{array}{l}
\Delta \mathbf{x}^{(k)} \\
\Delta \mathbf{y}^{(k)}
\end{array}\right]=\left[\begin{array}{c}
\alpha \mathbf{e}-\mathbf{x}^{(k)} \circ \mathbf{y}^{(k)} \\
\mathbf{0}
\end{array}\right]
$$

where

$$
\nabla_{\mathbf{x}} \mathbf{g}(\mathbf{x}, \mathbf{y})=\frac{1}{\sqrt{2}}\left[\begin{array}{cc}
x_{\mathbf{n}} & \mathbf{x}_{\mathbf{t}}^{T} \\
\mathbf{x}_{\mathbf{t}} & I_{2 \times 2} x_{\mathbf{n}}
\end{array}\right], \nabla_{\mathbf{y}} \mathbf{g}(\mathbf{x}, \mathbf{y})=\frac{1}{\sqrt{2}}\left[\begin{array}{cc}
y_{\mathbf{n}} & \mathbf{y}_{\mathbf{t}}^{T} \\
\mathbf{y}_{\mathbf{t}} & I_{2 \times 2} y_{\mathbf{n}}
\end{array}\right] .
$$

For the method to work, we need a feasible starting point. To this end, an artificial variable is introduced to transform the original complementarity problem of size $3 n$ into a complementarity problem of size $3 n+1$ with an obvious starting point. We introduce the additional variable $s \in \mathbb{R}_{+}$and a vector $\mathbf{d} \in \mathbb{R}^{3 n}$ and consider the complementarity problem

$$
\tilde{C} \ni \tilde{\mathbf{x}}=\left[\begin{array}{l}
\mathbf{x} \\
s
\end{array}\right], \tilde{C} \ni \tilde{\mathbf{y}}=\left[\begin{array}{l}
\overline{\mathbf{y}} \\
s
\end{array}\right]=\tilde{N} \tilde{\mathbf{x}}+\tilde{\mathbf{r}}=\left[\begin{array}{ll}
\bar{N} & \mathbf{d} \\
\mathbf{0} & 0
\end{array}\right]\left[\begin{array}{l}
\mathbf{x} \\
s
\end{array}\right]+\left[\begin{array}{l}
\mathbf{r} \\
1
\end{array}\right], 0=\tilde{\mathbf{x}}^{T} \tilde{\mathbf{y}}
$$

The central path of the new CCP is the zero set of

$$
f_{\text {cen }}(\tilde{\mathbf{x}}, \tilde{\mathbf{y}})=2(n+1) \log \frac{\mathbf{x}^{T} \mathbf{y} /(n+1)}{s^{\frac{1}{n+1}} \prod_{i=1}^{n}\left[2 \sqrt{\operatorname{det}\left(\mathbf{x}_{i}\right) \operatorname{det}\left(\mathbf{y}_{i}\right)}\right]^{\frac{1}{n+1}}} .
$$

Assume an initial feasible guess $\mathbf{x}^{(0)}$ for the original problem is given. For example, $\mathbf{x}_{i}^{(0)}=(1,0,0)^{T}$ obviously lies in int $\Upsilon$ for all $i=1, \ldots, n$. We now choose $s^{(0)}$ and $\mathbf{d}$ such that

$$
\overline{\mathbf{y}}=\tilde{\mathbf{y}}_{\{1, \ldots, 3 n\}}^{(0)}=\bar{N} \mathbf{x}^{(0)}+\mathbf{r}+s^{(0)} \mathbf{d} \in \operatorname{int} \Upsilon,
$$

Additionally, the parameters can be chosen in such a way that $\left(\tilde{\mathbf{x}}^{(0)}, \tilde{\mathbf{y}}^{(0)}\right)$ lies on a point $\alpha<0$ on the central path

$$
\tilde{\mathbf{x}}^{(0)} \circ \tilde{\mathbf{y}}^{(0)}=\alpha \mathbf{e}
$$

of the CCP. For all $i=1, \ldots, n$ it must hold

$$
\left[\begin{array}{c}
\sqrt{2} \alpha \\
0
\end{array}\right]=\frac{1}{\sqrt{2}}\left[\begin{array}{c}
x_{i n}^{(0)} \bar{y}_{i n}^{(0)}+\mathbf{x}_{i t}^{(0) T} \overline{\mathbf{y}}_{i t}^{(0)} \\
x_{i n}^{(0)} \overline{\mathbf{y}}_{i t}^{(0)}+\bar{y}_{i n}^{(0)} \mathbf{x}_{i t}^{(0)}
\end{array}\right] \Rightarrow \bar{y}_{i n}^{(0)}=\frac{2 \alpha}{x_{i n}^{(0)}-\frac{\left\|\mathbf{x}_{i t}^{(0)}\right\|^{2}}{x_{i n}^{(0)}}}, \overline{\mathbf{y}}_{i t}^{(0)}=-\left(\frac{\bar{y}_{i n}^{(0)}}{x_{i n}^{(0)}}\right) \mathbf{x}_{i t}^{(0)}
$$

For the last complementarity condition we have to make sure that

$$
s^{(0)} \cdot 1=s^{(0)}=\mathbf{x}_{i}^{(0) T} \overline{\mathbf{y}}_{i}^{(0)}=2 \alpha .
$$

This means that

$$
\mathbf{d}=\frac{1}{2 \alpha}\left(\overline{\mathbf{y}}^{(0)}-\bar{N} \mathbf{x}^{(0)}-\overline{\mathbf{r}}\right) .
$$


A search direction for Equation 75 is given by

$$
\left[\begin{array}{ccc}
\nabla_{\mathbf{x}} \mathbf{g}\left(\mathbf{x}^{(k)}, \mathbf{y}^{(k)}\right) & \nabla_{\mathbf{y}} \mathbf{g}\left(\mathbf{x}^{(k)}, \mathbf{y}^{(k)}\right) & \mathbf{0} \\
\bar{N} & -I & \mathbf{d} \\
\mathbf{0} & \mathbf{0} & 1
\end{array}\right]\left[\begin{array}{c}
\Delta \mathbf{x}^{(k)} \\
\Delta \mathbf{y}^{(k)} \\
\Delta s^{(k)}
\end{array}\right]=\left[\begin{array}{c}
\alpha \mathbf{e}-\mathbf{x}^{(k)} \circ \mathbf{y}^{(k)} \\
\mathbf{0} \\
2 \alpha-s^{(k)}
\end{array}\right]
$$

or equivalently by

$$
\begin{array}{r}
\Delta s^{(k)}=2 \alpha-s^{(k)}, \\
\tilde{\mathbf{A}} \Delta \mathbf{x}^{(k)}=\mathbf{b}, \\
\Delta \overline{\mathbf{y}}^{(k)}=\bar{N} \Delta \mathbf{x}^{(k)}+\Delta s^{(k)} \mathbf{d}
\end{array}
$$

where

$$
\begin{array}{r}
\tilde{\mathbf{A}}=\left[\nabla_{\mathbf{y}} \mathbf{g}\left(\mathbf{x}^{(k)}, \mathbf{y}^{(k)}\right)^{-1} \nabla_{\mathbf{x}} \mathbf{g}\left(\mathbf{x}^{(k)}, \mathbf{y}^{(k)}\right)+\bar{N}\right], \\
\mathbf{b}=\alpha\left(\mathbf{x}^{(k)}\right)^{-1}-\overline{\mathbf{y}}^{(k)}-\Delta s^{(k)} \mathbf{d} .
\end{array}
$$

and

$$
\mathbf{x}^{-1}=\frac{1}{\operatorname{det}(\mathbf{x})} J \mathbf{x}, J=\left[\begin{array}{cc}
1 & \mathbf{0} \\
\mathbf{0} & -I_{2 \times 2}
\end{array}\right] \in \mathbb{R}^{3 \times 3} .
$$

Since an optimal solution $\left(\tilde{\mathbf{x}}^{*}, \tilde{\mathbf{y}}^{*}\right)$ implies that $s^{*}=0$, it follows that $\left(\mathbf{x}^{*}, \overline{\mathbf{y}}^{*}\right)=\left(\mathbf{x}^{*}, \mathbf{y}^{*}\right)$ is optimal for the original CCP. During the iteration $s^{(k)}$ decreases and eventually $\mathbf{y}^{(k)}=$ $\overline{\mathbf{y}}^{(k)}-s^{(k)} \mathbf{d}$ is feasible. Then we can use the current iterate $\left(\mathbf{x}^{(k)}, \mathbf{y}^{(k)}\right)$ as a starting point for a feasible interior point for the original CCP.

After determining the direction of the step $(\Delta \mathbf{x}, \Delta \overline{\mathbf{y}})$, the next task is to identify the supremum of step sizes $\theta>0$ such that $\mathbf{x}+\theta \Delta \mathbf{x} \in$ int $\Upsilon$ and $\overline{\mathbf{y}}+\theta \Delta \overline{\mathbf{y}} \in$ int $\Upsilon$. As in [26], the maximum step size $\theta_{\max }$ for $\Delta \mathbf{x}$ and $\Delta \overline{\mathbf{y}}$ is determined by

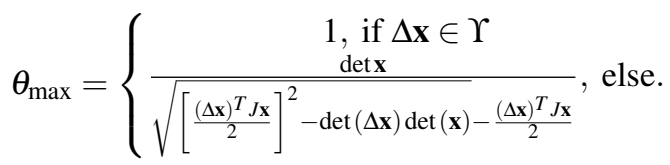

Finally, the convergence of the method can be improved substantially by rescaling the space in which the cone $\Upsilon$ lives at the beginning of each iteration using the Nesterov-Todd scaling scheme [26]. This is done by replacing Equation 83 with

$$
[P(\mathbf{w})+\bar{N}] \Delta \mathbf{x}^{(k)}=\mathbf{b},
$$

where

$$
P(\mathbf{w})=\mathbf{w} \mathbf{w}^{T}-\operatorname{det}(\mathbf{w}) J,
$$

and the scaling point $\mathbf{w} \in \mathbb{R}^{3}$ is

$$
\mathbf{w}=\frac{\mathbf{y}+\lambda J \mathbf{x}}{\sqrt{\mathbf{x}^{T} \mathbf{y}+2 \sqrt{\operatorname{det}(\mathbf{x}) \operatorname{det}(\mathbf{y})}}}
$$




\subsection{Termination criteria}

This section describes the stopping criteria used in the considered iterative methods. The residual, $r$, is defined as

$$
r=\arg \min \left(r_{p}, r_{d}, r_{c}\right)
$$

Residuals $r_{p}$ and $r_{d}$ are a measure of the feasibility of the primal and dual vectors

$$
r_{p}=\left\|\mathbf{f}_{p}\right\|_{\infty}, \mathbf{f}_{p}=\left[\ldots,-\min \left(0, \mu_{i} \gamma_{i, n}-\sqrt{\gamma_{i, u}^{2}+\gamma_{i, v}^{2}}\right), \ldots\right]^{T} \in \mathbb{R}^{N_{c}}
$$

and

$$
r_{d}=\left\|\mathbf{f}_{d}\right\|_{\infty}, \mathbf{f}_{d}=\left[\ldots,-\min \left(0, \frac{1}{\mu_{i}} \mathbf{y}_{i, n}-\sqrt{\mathbf{y}_{i, u}^{2}+\mathbf{y}_{i, v}^{2}}\right), \ldots\right]^{T} \in \mathbb{R}^{N_{c}}
$$

where $\vec{y}=\mathbf{N} \gamma+\mathbf{r}$. Residual $r_{c}$ is a measure of the worst violation of the actual complementarity condition as stated in Eq. 12

$$
r_{c}=\gamma^{T}(\mathbf{N} \gamma+\mathbf{r})
$$

In the PDIP method the iterates are not necessarily feasible, except in the limit as the algorithm converges. This means that we cannot easily evaluate a duality gap associated with step $k$ of the algorithm, as we do in the other methods. Instead we define a surrogate duality gap [38], for any $\gamma$ that satisfies $f(\gamma)<0$ and $\lambda \geq 0$, as

$$
\hat{\eta}(\gamma, \lambda)=-f(\gamma)^{T} \lambda
$$

The surrogate gap $\hat{\eta}$ would be the duality gap, if $\gamma$ were primal feasible and $\lambda$ were dual feasible.

\subsection{Sparse solver implementation}

Solving the linear system during step (9) of the PDIP algorithm in A.4 can present a large computational burden. A "split and parallelize" (SaP) approach can be used to solve linear systems $\mathbf{A x}=\mathbf{b}$ where $\mathbf{A}=\left(a_{i j}\right), 1 \leq i, j \leq N$, is dense banded or sparse. In the former case, the half-bandwidth is denoted $K$ and the matrix is assumed mildly diagonally dominant with coefficient $d$ :

$$
\left|a_{i i}\right| \geq d \sum_{j \neq i}\left|a_{i j}\right|, \forall i=1, \ldots, N
$$

$\mathrm{SaP}$ pursues one of four solution paths denoted $\mathrm{SaP}:: \mathrm{GPU}-\mathrm{D}_{\mathrm{d}}, \mathrm{SaP}:: \mathrm{GPU}-\mathrm{C}_{\mathrm{d}}, \mathrm{SaP}:: \mathrm{GPU}-\mathrm{D}_{\mathrm{s}}$, and SaP : : GPU- $\mathrm{C}_{\mathrm{s}}$, see Fig. 2][39]. When handling dense matrices, SaP : :GPU- $\mathrm{D}_{\mathrm{d}}$ splits the matrix $\mathbf{A}$ in $P$ partitions $\mathbf{A}_{i}$ that are independently LU-factored. The coupling between sub-blocks is ignored. On the other hand, SaP: : GPU- $\mathrm{C}_{\mathrm{d}}$ captures this coupling, in a fashion inspired by the SPIKE approach [40]. When handling sparse matrices, the implementation first pre-processes the coefficient matrix $\mathbf{A}$ to transform it from sparse to dense banded with heavy diagonal entries. To this end, we employ two reorderings: a diagonal boosting, and a bandwidth reduction. After potentially dropping 
off entries, the resulting matrix is treated as dense banded and solved pursuing one of two paths: with and without coupling between the diagonal blocks $\mathbf{A}_{i}$. Note that none of the four strategies leads to a direct solver owing to approximations embedded in the methodology. Consequently, solving $\mathbf{A x}=\mathbf{b}$ in SaP draws on a Krylovsubspace iterative solver that is preconditioned using the specific strategies associated with $\mathrm{SaP}:: \mathrm{GPU}-\mathrm{D}_{\mathrm{d}}, \mathrm{SaP}:: \mathrm{GPU}-\mathrm{C}_{\mathrm{d}}$, etc. The entire solution approach is GPU-parallel, with the exception of the matrix reorderings, which are implemented using a hybrid CPU-GPU approach [39].

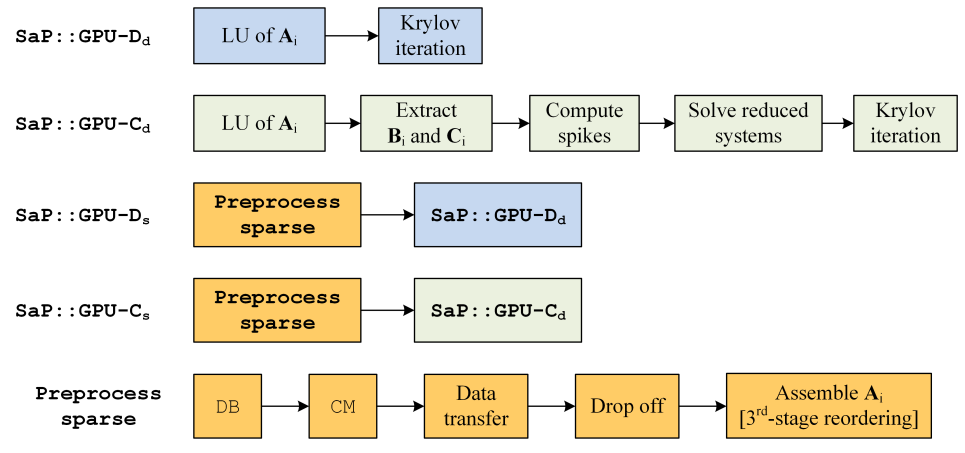

Figure 2: Computational flow for SaP.

\section{Numerical experiments}

\subsection{Filling tests}

The first numerical experiment studies a model of spherical bodies falling into a container under gravity, the time evolution of which can be seen in Fig. 3. The goal of this study was to see how the solver tolerance effects the physical results of the simulation. Several statistics were monitored, such as the computation time, number of iterations performed at each step, and contact force on the container. In this case, there are 1000 spheres, each with a radius $r=1 \mathrm{~m}$, mass $m=1 \mathrm{~kg}$, and friction coefficient $\mu=0.25$. The system had a gravitational acceleration in the vertical direction $g=9.81 \mathrm{~m} \mathrm{~s}^{-1}$. The simulation was run for $3 \mathrm{~s}$ with a time step $h=0.01 \mathrm{~s}$ and a varying solver tolerance.

The results in Fig. 4 show the average percent error in contact force that the container experiences during the last second of an eleven second dynamics simulation. Since the only external force that is acting on the bodies is due to gravity, the average contact force that the container experiences should be equal to the total weight of the spheres and an analytical solution is easily determined. There are two conclusions that can be drawn from this study. First, as the solver tolerance is decreased, or "tightened", the average contact force approaches the analytical result. Second, the average error as a function of the residual is relatively independent of the solver. In the case of the filling test, the solver residual is roughly proportional to the average physical error.

The efficiency of the solver refers to how the number of iterations required to reach a tolerance change as a function of the solver residual. Several conclusions can be 


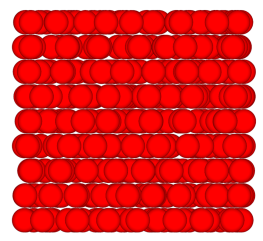

$\mathrm{t}=0 \mathrm{~s}$

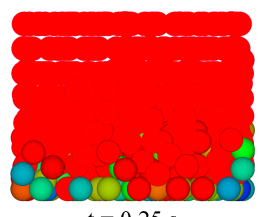

$\mathrm{t}=0.25 \mathrm{~s}$

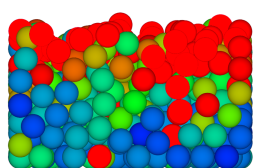

$\mathrm{t}=0.5 \mathrm{~s}$

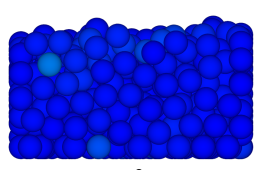

$\mathrm{t}=3 \mathrm{~s}$

Figure 3: A simulation of 1000 spherical bodies falling into a container due to gravity.

drawn from the results of this study, shown in Fig. 5. In general, as the tolerance is decreased, the number of iterations required by all of the solvers increases. Based on the filling test, the PGJ solver requires the largest amount of iterations, although the PGS solver is comparable. In general, the SCIP and P-SCIP solvers require the least amount of iterations. It should be noted that the first-order solvers require less iterations than the second-order solvers for large residuals, although solvers ran at such a large tolerance would produce nonphysical behavior. In the case of the filling test, the spheres would never quite settle in the container (i.e. there would be a large amount of "chatter"). Finally, although the number of iterations is important to consider, it does not completely depict the efficiency of the solver. It is important to consider the execution time, as studied in $\$ 4.2$

\subsection{Scaling Analysis}

A scaling analysis was performed to study the effect of the number of bodies on the convergence of the solvers. Much like the previous sections, this analysis studies a model of spherical bodies falling into a container under gravity. Several statistics were monitored, such as the computation time and number of iterations performed at each step. The scaling analysis varied the number of spheres that were being dropped into the container from 160 to 16000 spheres, each with a radius $r=1 \mathrm{~m}$, mass $m=1 \mathrm{~kg}$, and friction coefficient $\mu=0.25$. The systems each had a gravitational acceleration in the vertical direction $g=9.81 \mathrm{~m} \mathrm{~s}^{-1}$. The simulation was run for $11 \mathrm{~s}$ with a time step $h=0.01 \mathrm{~s}$ and a solver tolerance $\tau=1 \times 10^{-3}$. The results correspond to the last second of the simulation.

The average number of iterations required for the solvers to reach a tolerance $\tau=1 \times 10^{-3}$ is shown in Fig. 6 . Much like in $\$ 4.1$, the second-order solvers require significantly fewer iterations than the first-order solvers. For example, for the case of 16000 bodies being dropped into the container, the SCIP solvers require approximately $50 \mathrm{X}$ fewer iterations than the APGD solver and $60000 \mathrm{X}$ fewer iterations than the PGJ solver.

As mentioned in 4.1 , the average number of iterations does not give a complete depiction of the solver performance. The total execution time to solve the final second of the filling simulation as a function of the number of bodies is shown in Fig.7 7 Based on this data, the APGD solver the fastest solver for large numbers of bodies in terms of computational time. Additionally, the computational time for the APGD solver scales 


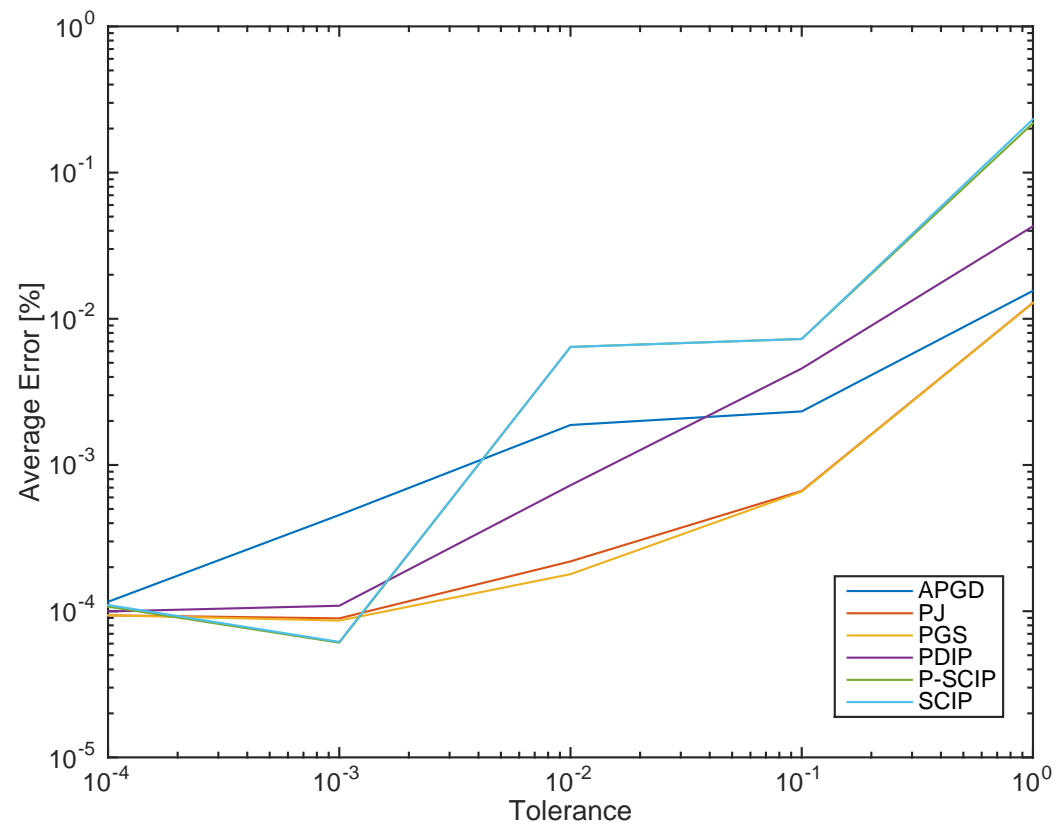

Figure 4: The average contact force that the container experienced for the last second of simulation in the filling test.

at a slower rate than the second-order solvers. Interestingly, for simulations where the number of bodies is low (i.e. less than 500), the P-SCIP solver is preferable in terms of execution time.

\section{Validation}

The second numerical experiment, which served as a validation of the contact method and solvers, involved the drafting test, shown in Fig. 8. The drafting test is comprised of a rectangular blade that moves through a trench filled with granular material. In this case, there are 21638 spherical bodies with a density $\rho=2500 \mathrm{~kg} \mathrm{~cm}^{-3}$, a friction coefficient $\mu=0.25$, and a randomly distributed radius between 0.008 and $0.016 \mathrm{~m}$. The blade has a width of $0.2 \mathrm{~m}$ and moves with a constant horizontal speed of $0.2 \mathrm{~m} \mathrm{~s}^{-1}$ at an initial depth of $0.2 \mathrm{~m}$. The simulation was solved for $3 \mathrm{~s}$ of simulation time using the APGD solver with a time step $h=0.001 \mathrm{~s}$ and a tolerance $\tau=1 \times 10^{-4}$ $\mathrm{N}$. At $t=3 \mathrm{~s}$, the force that the blade experienced was considered to have reached a steady value and the system state was exported to a file. The system state was imported into each solver described in $\$ 3$ and the contact force and residual were recorded at each iteration during the solution of a single time step in Fig. 9.

The force that the blade experienced is plotted as a function of the residual for a single time step during steady-state operation in Fig. 9a. Based on this data, the draft 


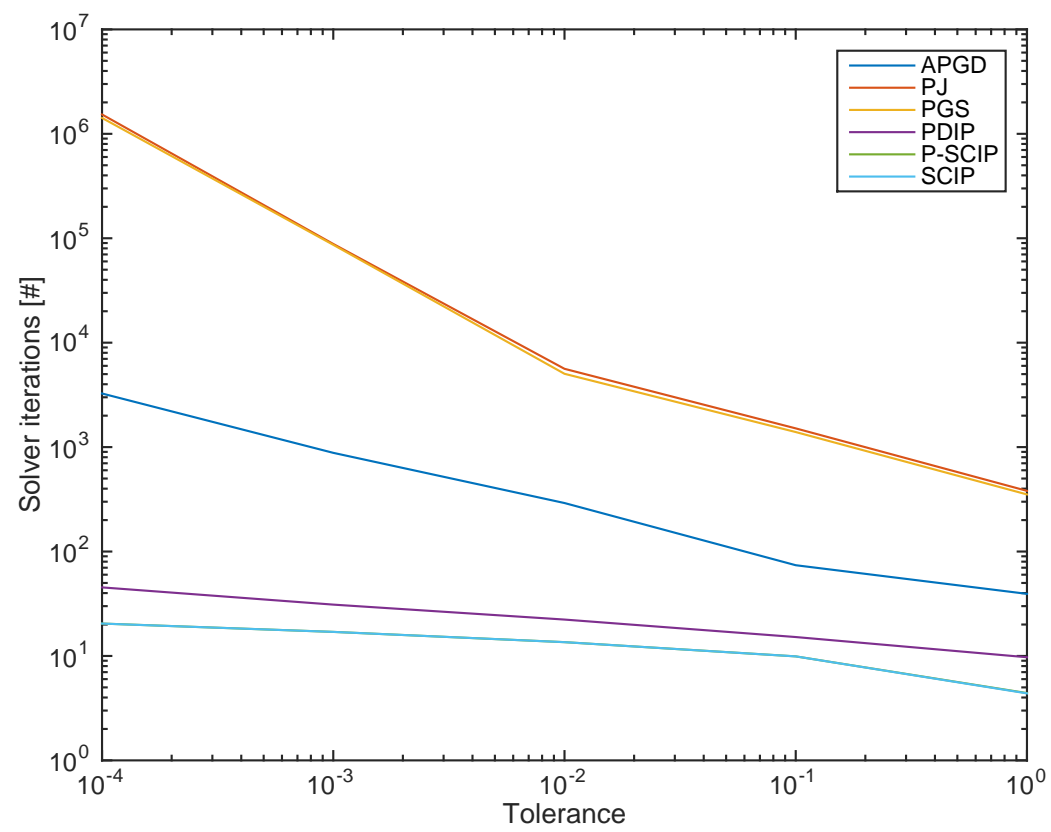

Figure 5: The average number of solver iterations required to reach a specified tolerance for the final second of an eleven second filling operation.

force for a given residual is independent of the solver, demonstrating that the residual definition (described in $\$ 3.3$ is an accurate indication of the convergence across the different solvers. Conversely, the number of iterations required to obtain a given residual, shown in Fig. $9 \mathrm{~b}$, is quite dependent on the solver. The interior point solvers require much fewer iterations than the first-order solvers, which is consistent with the filling results in $\$ 4$.

\section{Conclusions}

The computational bottleneck of a widely-used DVI methodology for simulating frictional contact dynamics is tied to the solution of a conically constrained convex optimization problem. This contribution compares two classes of methods aimed at efficiently solving this optimization problem. We considered three first-order methods: projected Gauss-Jacobi (PGJ), projected Gauss-Seidel (PGS), and accelerated projected gradient descent (APGD); and two second-order ones: a symmetric cone interior point (SCIP) method and a primal-dual interior point (PDIP) method. The latter methods rely on a Newton step to identify the descent direction and a line search to compute the step size. All five methods draw on GPU computing; the second order methods also employ a sparse parallel GPU solver in their Newton step. Filling, drafting and scaling numerical experiments were carried out to evaluate the performance 


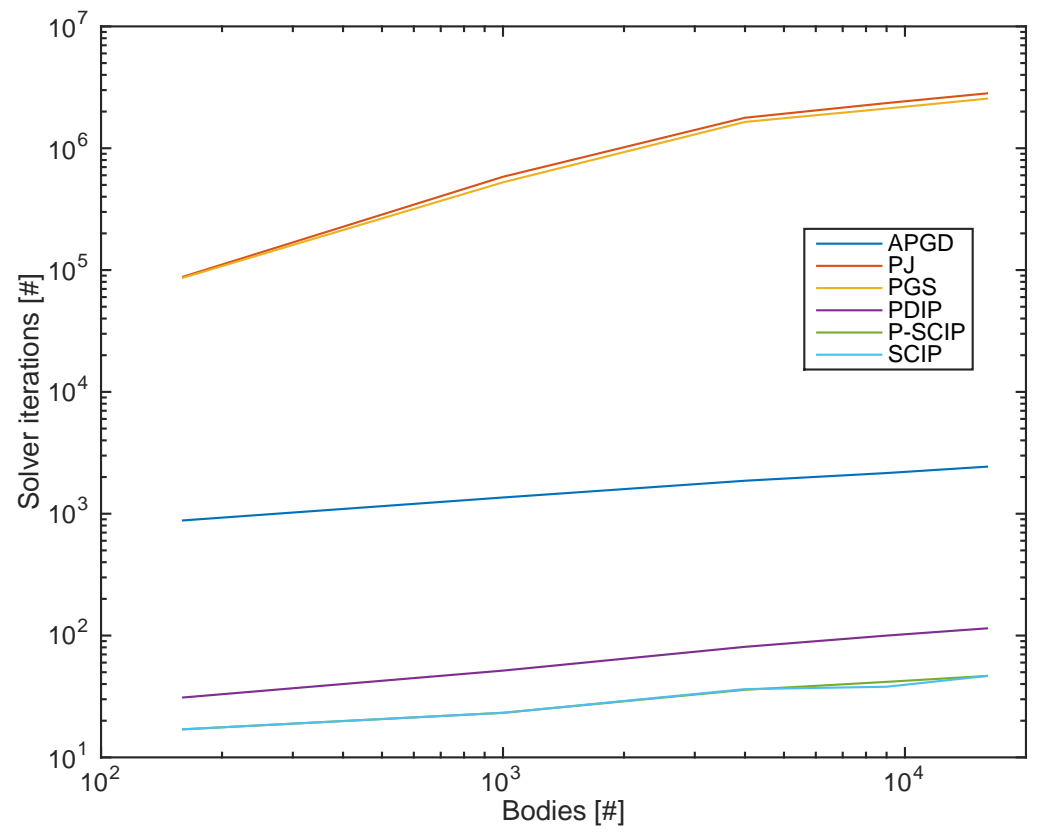

Figure 6: The average number of solver iterations to solve a time step during the final second of the filling simulation.

of the five methods in terms of convergence rate, accuracy, and computational cost. Attention was paid to providing a fair comparison across the different solvers. To that end, all numerical experiments were performed in the same open source code [41, 42] modified to host the five methods of interest.

The numerical experiments reported here as well as other terramechanics tests [43] led us to conclude that for packed-body scenarios such as granular dynamics or terrain modeling, APGD is the most efficient solver; i.e., it reaches a certain level of accuracy in a shorter amount of time. For problems that involve less than 500 bodies, the second-order methods perform better. Overall, the interior point methods require much fewer iterations than the first-order solvers with the SCIP method requiring the fewest. Additionally, the SCIP method does not appear to require more iterations as the problem difficulty is increased, as shown in the compaction test. The interior point methods are computationally more expensive due in large part to the linear system that must be solved at each iteration of the interior point algorithm. Finally, while PDIP and SCIP are relatively similar in terms of performance, for the first-order methods there is a wide efficiency gap between the performance of APGD on the one hand, and PJ and PGS on the other hand. In fact, if we exclude APGD from this comparison, our scaling analysis suggests that PJ and PGS cannot surpass SCIP in terms of efficiency. It should be pointed out that a GPU implementation like the one pursued here favors first-order methods and this 500 threshold value might go up if one switches to CPU parallel com- 


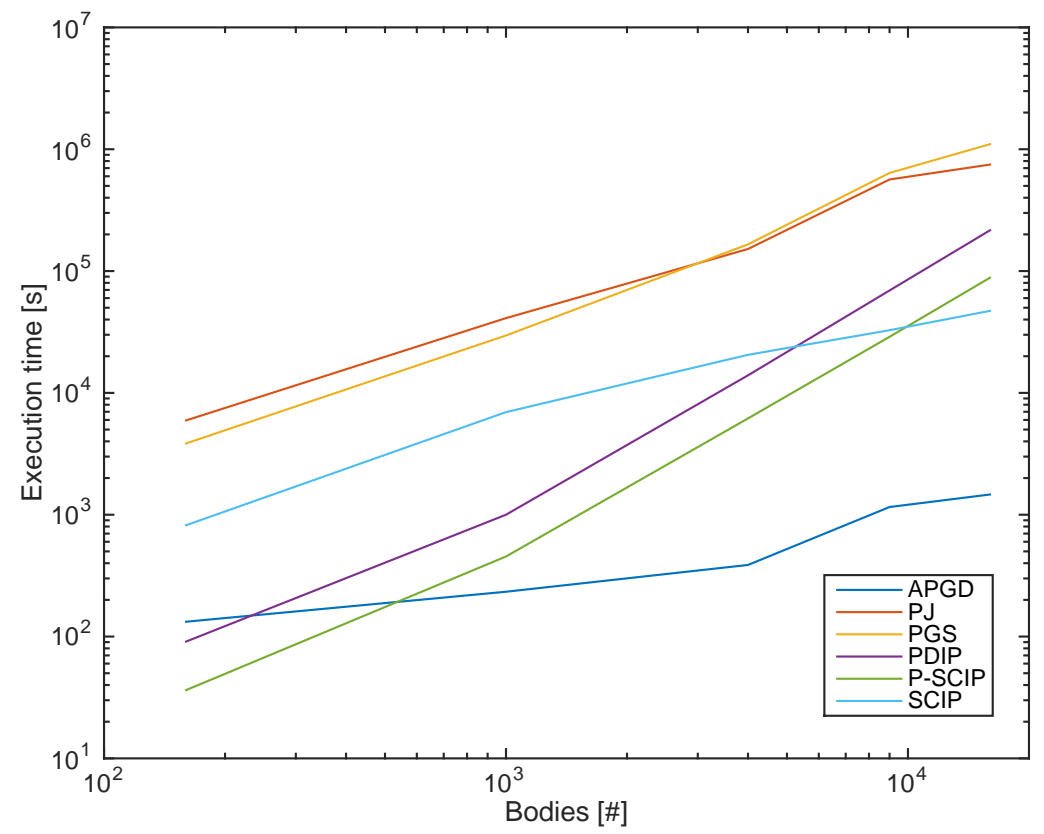

Figure 7: The total execution time to solve the final second of the filling simulation.

puting. Indeed, GPU computing has an edge for larger problems where the overhead of GPU parallelization is overshadowed by the sheer amount of computation and data movement associated with the solution. However, this overhead is not amortized for problem sizes in the range of 500 bodies, and a switch to a multi-core CPU solution will likely move the above threshold value at which second order methods stop paying off to larger body counts.

\section{Acknowledgments}

Financial support was provided by Army Research Office award W911NF-12-10395 and National Science Foundation award CMMI-GOALI-1362583.

\section{References}

[1] P. Richard, M. Nicodemi, R. Delannay, P. Ribiere, D. Bideau, Slow relaxation and compaction of granular systems, Nature Materials 4 (2) (2005) 121-128.

[2] D. Stewart, Existence of solutions to rigid body dynamics and the Painlevé paradoxes, C. R. Acad. Sci. Paris 325 (1997) 689-693.

[3] E. J. Haug, Computer-Aided Kinematics and Dynamics of Mechanical Systems Volume-I, Prentice-Hall, Englewood Cliffs, New Jersey, 1989. 


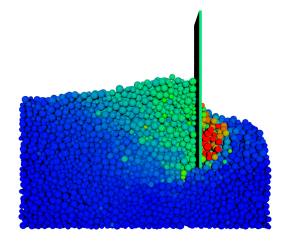

(a)

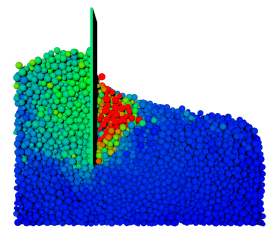

(b)

Figure 8: A DEM simulation of a blade moving through granular material at the initial (left) and final (right) configurations.

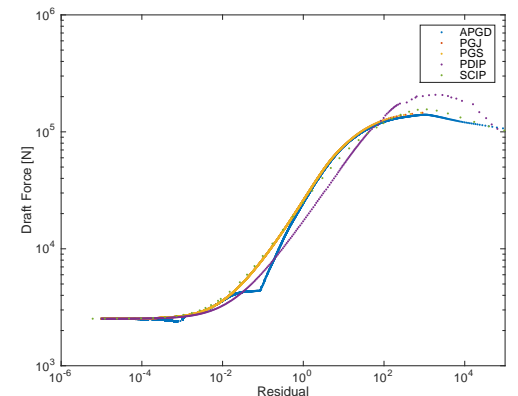

(a)

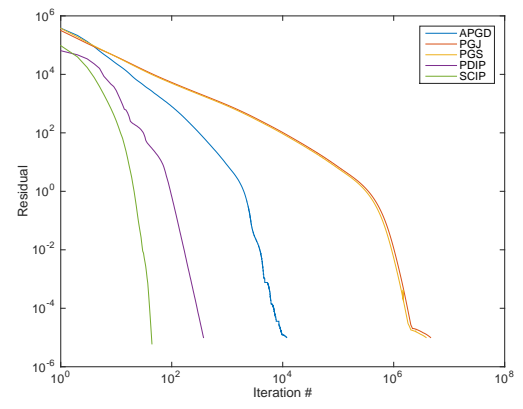

(b)

Figure 9: The draft force that the blade experiences as a function of the residual (left) and as a function of the iteration number (right) for a single time step during steady-state operation.

[4] A. A. Shabana, Computational Dynamics, John Wiley \& Sons, 1994.

[5] D. E. Stewart, Rigid-body dynamics with friction and impact, SIAM Review 42(1) (2000) 3-39.

[6] D. E. Stewart, J. C. Trinkle, An implicit time-stepping scheme for rigid-body dynamics with inelastic collisions and Coulomb friction, International Journal for Numerical Methods in Engineering 39 (1996) 2673-2691.

[7] M. Anitescu, F. A. Potra, Formulating dynamic multi-rigid-body contact problems with friction as solvable linear complementarity problems, Nonlinear Dynamics 14 (1997) 231-247.

[8] E. Hairer, G. Wanner, Solving Ordinary Differential Equations II: Stiff and Differential-Algebraic Problems, Springer, 1996.

[9] M. Anitescu, Optimization-based simulation of nonsmooth rigid multibody dynamics, Mathematical Programming 105 (1) (2006) 113-143. doi:http://dx. doi.org/10.1007/s10107-005-0590-7 
[10] D. Stewart, Convergence of a time-stepping scheme for rigid body dynamics and resolution of Painlevé's problems, Archive Rational Mechanics and Analysis 145 (3) (1998) 215-260.

[11] T. Heyn, On the Modeling, Simulation, and Visualization of Many-Body Dynamics Problems with Friction and Contact, PhD thesis, Department of Mechanical Engineering, University of Wisconsin-Madison, http://sbel.wisc.edu/ documents/TobyHeynThesis_PhDfinal.pdf (2013).

[12] F. Bertails-Descoubes, F. Cadoux, G. Daviet, V. Acary, A nonsmooth Newton solver for capturing exact Coulomb friction in fiber assemblies, ACM Transactions on Graphics (TOG) 30 (1) (2011) 6.

[13] G. Daviet, F. Bertails-Descoubes, L. Boissieux, A hybrid iterative solver for robustly capturing coulomb friction in hair dynamics, in: ACM Transactions on Graphics (TOG), Vol. 30 (6), ACM, 2011, p. 139.

[14] V. Acary, F. Cadoux, C. Lemarechal, J. Malick, A formulation of the linear discrete coulomb friction problem via convex optimization, ZAMM-Journal of Applied Mathematics and Mechanics/Zeitschrift für Angewandte Mathematik und Mechanik 91 (2) (2011) 155-175.

[15] O. Bonnefon, G. Daviet, Quartic formulation of Coulomb 3d frictional contact technical report rt-0400, inria, Tech. rep., INRIA - Laboratoire Jean Kuntzmann, Grenoble (2011).

[16] J. J. Moreau, M. Jean, Numerical treatment of contact and friction: The contact dynamics method, in: Proceedings of the Third Biennial Joint Conference on Engineering Systems and Analysis, Montpellier, France, 1996, pp. 201-208.

[17] M. Anitescu, G. D. Hart, A constraint-stabilized time-stepping approach for rigid multibody dynamics with joints, contact and friction, International Journal for Numerical Methods in Engineering 60(14) (2004) 2335-2371.

[18] C. Glocker, F. Pfeiffer, An LCP-approach for multibody systems with planar friction, in: Proceedings of the CMIS 92 Contact Mechanics Int. Symposium, Lausanne, Switzerland, 2006, pp. 13-20.

[19] A. Tasora, D. Negrut, M. Anitescu, Large-scale parallel multi-body dynamics with frictional contact on the Graphical Processing Unit, Journal of Multi-body Dynamics 222 (4) (2008) 315-326.

[20] T. M. Preclik, K. Iglberger, U. Rüde, Iterative rigid multibody dynamics, in: Proceeding of Multibody Dynamics ECCOMAS Thematic Conference, 2009, pp. $1-17$.

[21] Z. Shojaaee, M. R. Shaebani, L. Brendel, J. Toeroek, D. E. Wolf, An adaptive hierarchical domain decomposition method for parallel contact dynamics simulations of granular materials, Journal of Computational Physics 231 (2) (2012) 612-628. doi: $\{10.1016 / j \cdot j c p .2011 .09 .024\}$. 
[22] R. Tonge, F. Benevolenski, A. Voroshilov, Mass splitting for jitter-free parallel rigid body simulation, ACM Transactions on Graphics (TOG) 31 (4) (2012) 105.

[23] T. Heyn, M. Anitescu, A. Tasora, D. Negrut, Using Krylov subspace and spectral methods for solving complementarity problems in many-body contact dynamics simulation IJNME 95 (7) (2013) 541-561. doi:10.1002/nme.4513. URL http://dx.doi.org/10.1002/nme.4513

[24] H. Mazhar, T. Heyn, A. Tasora, D. Negrut, Using Nesterov's method to accelerate multibody dynamics with friction and contact, ACM Trans. Graph. 34 (3).

[25] L. Fang, A Primal-Dual Interior Point Method for Solving Multibody Dynamics Problems with Frictional Contact, Master's thesis, University of WisconsinMadison, Madison, WI, USA (2014).

[26] J. Kleinert, Simulating granular material using nonsmooth time-stepping and a matrix-free interior point method, Ph.D. thesis, University of Kaiserslautern (2015).

[27] M. Anitescu, A. Tasora, An iterative approach for cone complementarity problems for nonsmooth dynamics, Computational Optimization and Applications 47 (2) (2010) 207-235. doi:10.1007/s10589-008-9223-4.

[28] A. Tasora, M. Anitescu, A matrix-free cone complementarity approach for solving large-scale, nonsmooth, rigid body dynamics, Computer Methods in Applied Mechanics and Engineering 200 (5-8) (2011) 439-453. doi:doi:10.1016/j. cma.2010.06.030.

[29] D. Negrut, A. Tasora, M. Anitescu, H. Mazhar, T. Heyn, A. Pazouki, Solving large multi-body dynamics problems on the GPU, GPU Gems Vol. 4 (2011) 269-280.

[30] Y. Nesterov, A method of solving a convex programming problem with convergence rate $\mathscr{O}\left(1 / k^{2}\right)$, in: Soviet Mathematics Doklady, Vol. 27(2), 1983, pp. 372376.

[31] A. Cauchy, Méthode générale pour la résolution des systemés d'équations simultanées, Comp. Rend. Sci. Paris 25 (1847) (1847) 536-538.

[32] D. Bertsekas, On the Goldstein-Levitin-Polyak gradient projection method, Automatic Control, IEEE Transactions on 21 (2) (1976) 174-184.

[33] A. Nemirovsky, D. B. Yudin, Problem complexity and method efficiency in optimization., John Wiley \& Sons, 1983.

[34] Y. Nesterov, Introductory lectures on convex optimization: A basic course, Vol. 87, Springer, 2003.

[35] A. Beck, M. Teboulle, A fast iterative shrinkage-thresholding algorithm for linear inverse problems, SIAM Journal on Imaging Sciences 2 (1) (2009) 183-202. 
[36] S. R. Becker, E. J. Candès, M. C. Grant, Templates for convex cone problems with applications to sparse signal recovery, Mathematical Programming Computation 3 (3) (2011) 165-218.

[37] B. O’Donoghue, E. Candes, Adaptive restart for accelerated gradient schemes, ArXiv e-prints arXiv: 1204.3982

[38] S. Boyd, L. Vandenberghe, Convex optimization, Cambridge University Press, 2004.

[39] A. Li, R. Serban, D. Negrut, Analysis of a splitting approach for the parallel solution of linear systems on GPU cards, SIAM Journal of Scientific Computing - in press $00(0)$ (2017) 0.

[40] E. Polizzi, A. Sameh, A parallel hybrid banded system solver: the SPIKE algorithm, Parallel Computing 32 (2) (2006) 177-194.

[41] A. Tasora, R. Serban, H. Mazhar, A. Pazouki, D. Melanz, J. Fleischmann, M. Taylor, H. Sugiyama, D. Negrut, Chrono: An open source multi-physics dynamics engine, in: T. Kozubek (Ed.), High Performance Computing in Science and Engineering - Lecture Notes in Computer Science, Springer, 2016, pp. 19-49.

[42] Project Chrono, Chrono: An Open Source Framework for the Physics-Based Simulation of Dynamic Systems, http://projectchrono.org, accessed: 201603-07.

[43] D. Melanz, Physics-based contact using the complementarity approach for discrete element applications in vehicle mobility and terramechanics, PhD thesis, http://sbel.wisc.edu/documents/thesisPhD-DanielMelanz.pdf, University of Wisconsin-Madison (2016).

\section{Appendices}

\section{A. Algorithms of iterative methods}

Description of algorithms used

\section{A.1. Projected Gauss-Jacobi method}

Algorithm Jacobi(N, $\left.\mathbf{r}, \tau, N_{\max }, \gamma_{0}\right)$

(1) for $k:=0$ to $N_{\max }$

(2) $\quad \hat{\gamma}_{(k+1)}=\Pi_{\mathscr{K}}\left(\gamma_{(k)}-\omega \mathbf{B}\left(\mathbf{N} \gamma_{(k)}+\mathbf{r}\right)\right)$

(3) $\quad \gamma_{(k+1)}=\lambda \hat{\gamma}_{(k+1)}+(1-\lambda) \gamma_{(k)}$

(4) $\quad r=r\left(\gamma_{(k+1)}\right)$

(5) $\quad$ if $r<\tau$

(6) break

(7) endfor

(8) return Value at time step $t^{(l+1)}, \gamma^{(l+1)}:=\gamma_{(k+1)}$. 


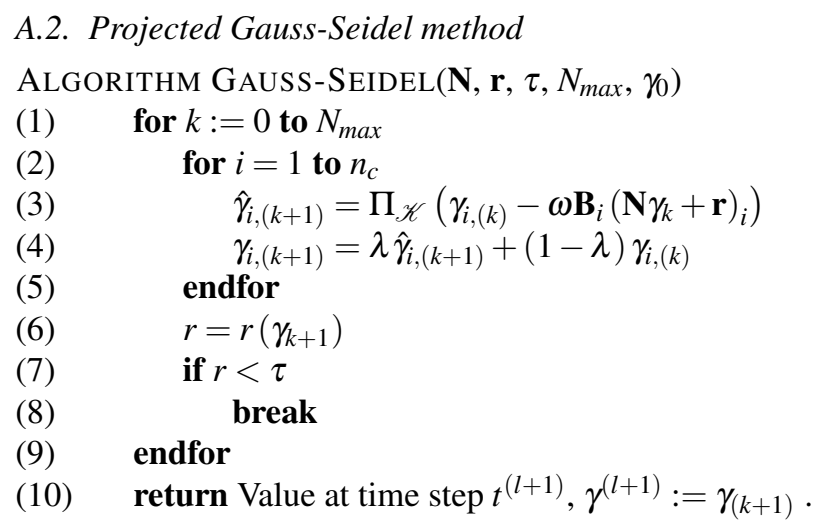




\section{A.3. Accelerated projected gradient descent method}

ALGORITHM APGD(N, $\left.\mathbf{r}, \tau, N_{\max }\right)$

(1) $\gamma_{0}=\mathbf{0}_{n_{c}}$

(2) $\hat{\gamma}_{0}=\mathbf{1}_{n_{c}}$

(3) $\mathbf{y}_{0}=\gamma_{0}$

(4) $\theta_{0}=1$

(5) $\quad L_{k}=\frac{\left\|\mathbf{N}\left(\gamma_{0}-\hat{\gamma}_{0}\right)\right\|_{2}}{\left\|\gamma_{0}-\hat{\gamma}_{0}\right\|_{2}}$

(6) $t_{k}=\frac{1}{L_{k}}$

(7) for $k:=0$ to $N_{\max }$

(8) $\mathbf{g}=\mathbf{N y}_{k}+\mathbf{r}$

(9) $\quad \gamma_{k+1}=\Pi_{\mathscr{K}}\left(\mathbf{y}_{k}-t_{k} g\right)$

(10) $\quad$ while $\frac{1}{2} \gamma_{k+1}^{T} \mathbf{N} \gamma_{k+1}+\gamma_{k+1}^{T} \mathbf{r} \geq \frac{1}{2} \mathbf{y}_{k}^{T} \mathbf{N} \mathbf{y}_{k}+\mathbf{y}_{k}^{T} \mathbf{r}+\mathbf{g}^{T}\left(\gamma_{k+1}-\mathbf{y}_{k}\right)+\frac{1}{2} L_{k} \| \gamma_{k+1}-$ $\mathbf{y}_{k} \|_{2}^{2}$

$L_{k}=2 L_{k}$

$t_{k}=\frac{1}{L_{k}}$

$\gamma_{k+1} \stackrel{L_{k}}{=} \Pi_{\mathscr{K}}\left(\mathbf{y}_{k}-t_{k} g\right)$

$$
\text { endwhile }
$$

$$
\begin{aligned}
& \theta_{k+1}=\frac{-\theta_{k}^{2}+\theta_{k} \sqrt{\theta_{k}^{2}+4}}{2^{2}} \\
& \beta_{k+1}=\theta_{k} \frac{1-\theta_{k}}{\theta_{k}^{2}+\theta_{k+1}} \\
& \mathbf{y}_{k+1}=\gamma_{k+1}+\beta_{k+1}\left(\gamma_{k+1}-\gamma_{k}\right) \\
& r=r\left(\gamma_{k+1}\right) \\
& \text { if } r<\varepsilon_{\min } \\
& \quad r_{\min }=r \\
& \hat{\gamma}=\gamma_{k+1} \\
& \text { endif } \\
& \text { if } r<\tau \\
& \quad \text { break } \\
& \text { endif } \\
& \text { if } \mathbf{g}^{T}\left(\gamma_{k+1}-\gamma_{k}\right)>0 \\
& \quad \mathbf{y}_{k+1}=\gamma_{k+1} \\
& \quad \theta_{k+1}=1 \\
& \text { endif } \\
& L_{k}=0.9 L_{k} \\
& t_{k}=\frac{1}{L_{k}} \\
& \text { endfor } \\
& \text { return Value at time step } t_{l+1}, \gamma^{l+1}:=\hat{\gamma} .
\end{aligned}
$$




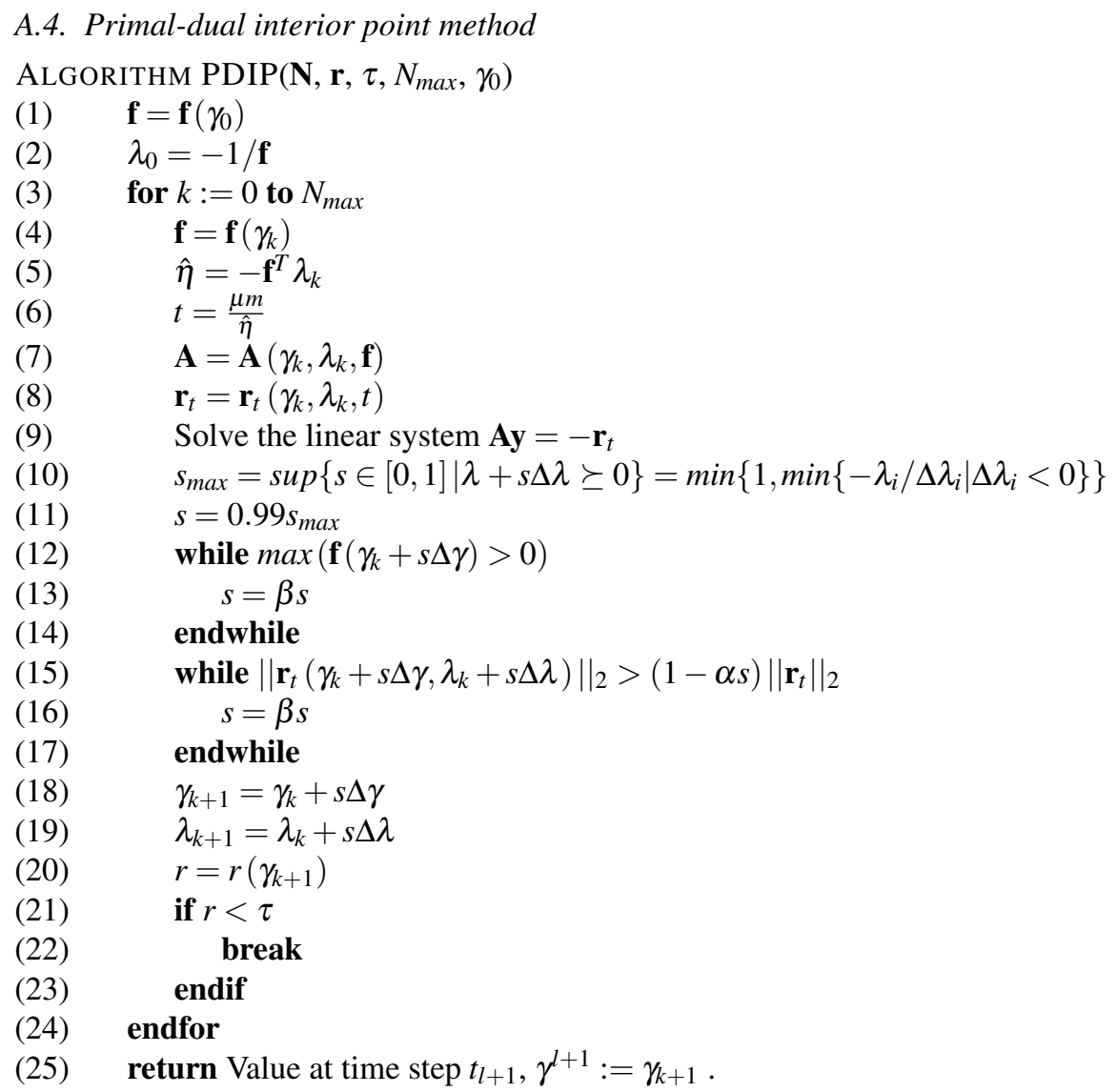




\section{A.5. Symmetric cone interior point method}

\section{Algorithm SCIP(N, $\left.\mathbf{r}, \tau, N_{\max }, \gamma_{0}\right)$}

(1) $\bar{N}=T_{y} N T_{x}^{-1}$

(2) $\overline{\mathbf{r}}=T_{y} \mathbf{r}$

(3) Guess a feasible initial $\mathbf{x}^{(0)}=T_{x} \gamma^{(0)}$

(4) Construct $\mathbf{d}$ and $s^{(0)}$ as in Equations $79-81$

(5) $\quad \overline{\mathbf{y}}^{(0)}=\bar{N} \overline{\mathbf{x}}^{(0)}+\mathbf{r}+s^{(0)} \mathbf{d}$

(6) Set feasible $=0$

(7) for $k:=0$ to $N_{\max }$

(8) Calculate $P(\mathbf{w})$ using Equations 88 and 89

(9) $\quad A=P(\mathbf{w})+\bar{N}$

(10) $\Delta s^{(k)}=(1-$ feasible $) \cdot\left(2 \alpha-s^{(k)}\right)$

$$
\mathbf{b}=\alpha\left(\mathbf{x}^{(k)}\right)^{-1}-\overline{\mathbf{y}}^{(k)}-\Delta s^{(k)} \mathbf{d}
$$

Solve $A \Delta \mathbf{x}=\mathbf{b}$

Calculate stepping length $\theta$ using Equation 86

$$
\begin{aligned}
& \mathbf{x}^{(k+1)}=\mathbf{x}^{(k)}+\theta \Delta \mathbf{x} \\
& \mathbf{y}^{(k+1)}=\mathbf{y}^{(k)}+\boldsymbol{\theta}\left(\bar{N} \Delta \mathbf{x}+\Delta s^{(k)} \mathbf{d}\right)
\end{aligned}
$$$$
s^{(k+1)}=s^{(k)}+\theta \Delta s^{(k)}
$$

if feasible $=0$ and $\overline{\mathbf{y}}^{(k+1)}-s^{(k+1)} \mathbf{d} \in \operatorname{int} \Upsilon$ then $\overline{\mathbf{y}}^{(k+1)}=\overline{\mathbf{y}}^{(k+1)}-s^{(k+1)} \mathbf{d}$ $s^{(k+1)}=0$

feasible $=1$

$\alpha=\beta \frac{\mathbf{x}^{(k) T} \overline{\mathbf{y}}^{(k)}}{2 N_{c}}$ for some $\beta \in(0,1]$

$\gamma^{(k+1)}=T_{x}^{-1} \mathbf{x}^{(k+1)}$

if $r<\tau$

break

endif

endfor

return Value at time step $t_{l+1}, \gamma^{l+1}:=\gamma^{(k+1)}$.

\section{B. Positive Definiteness Result}

We set out here to prove that the matrix $\mathbf{N}+\hat{\mathbf{M}}-\mathbf{B D}^{-1} \mathbf{C}$ is positive definite. To this end, let $\hat{\mathbf{D}}=\hat{\mathbf{M}}-\mathbf{B} \mathbf{D}^{-1} \mathbf{C}^{T}$, where $\hat{\mathbf{D}}$ is a block diagonal matrix, with the $i^{\text {th }}$ block expressed as

$$
\hat{\mathbf{D}}_{i}=\left[\begin{array}{lll}
-\mu_{i}^{2} \lambda_{i}-\mu_{i}^{4} \gamma_{i, n}^{2} \frac{\lambda_{i}}{f_{i}}-\frac{\lambda_{i+N_{c}}}{f_{i+N_{c}}} & \mu_{i}^{2} \gamma_{i, n} \gamma_{i, u} \frac{\lambda_{i}}{f_{i}} & \mu_{i}^{2} \gamma_{i, n} \gamma_{i, w} \frac{\lambda_{i}}{f_{i}} \\
& \lambda_{i}-\gamma_{i, u}^{2} \frac{\lambda_{i}}{f_{i}} & -\gamma_{i, u} \gamma_{i, w} \frac{\lambda_{i}}{f_{i}} \\
\operatorname{sym} & & \lambda_{i}-\gamma_{i, w}^{2} \frac{\lambda_{i}}{f_{i}}
\end{array}\right] .
$$

First, we will show that $\hat{\mathbf{D}}$ is positive definite. The proof uses the Sylvester's criterion, which states that a matrix $\hat{\mathbf{D}} \in \mathbb{R}^{n \times n}$ is positive definite if and only if all the principal minors $\Delta_{p}(\hat{\mathbf{D}})>0, \quad \forall p=1, \ldots, n$. For simplicity, subscript $i$ is dropped, $\lambda_{i}$ and $\lambda_{i+N_{c}}$ are denoted as $\lambda_{1}$ and $\lambda_{2} ; f_{i}$ and $f_{i+N_{c}}$ are denoted as $f_{1}$ and $f_{2}$. 


\section{Proof:}

We compute the three principal minors of $\mathbf{D}_{i}$.

$$
\Delta_{1}=-\mu^{2} \lambda_{1}-\mu^{4} \gamma_{n}^{2} \frac{\lambda_{1}}{f_{1}}-\frac{\lambda_{2}}{f_{2}}
$$

for which, $-\lambda_{2} / f_{2}>0$, since $\lambda_{i}>0$ and $f_{i}<0$. Then,

$$
-\mu^{2} \lambda_{1}-\mu^{4} \gamma_{n}^{2} \frac{\lambda_{1}}{f_{1}}=\frac{-\mu^{2} \lambda_{1}}{f_{1}}\left(f_{1}+\mu^{2} \gamma_{n}^{2}\right)
$$

where $-\mu^{2} \lambda_{1} / f_{1}>0$, and

$$
f_{1}+\mu^{2} \gamma_{n}^{2}=\frac{1}{2}\left(\gamma_{u}^{2}+\gamma_{v}^{2}-\mu^{2} \gamma_{n}^{2}\right)+\mu^{2} \gamma_{n}^{2}=\frac{1}{2}\left(\gamma_{u}^{2}+\gamma_{v}^{2}+\mu^{2} \gamma_{n}^{2}\right)>0
$$

and therefore, $\Delta_{1}>0$. For the next minor, we have

$$
\begin{aligned}
\Delta_{2} & =\operatorname{det}\left(\left[\begin{array}{ll}
-\mu^{2} \lambda_{1}-\mu^{4} \gamma_{n}^{2} \frac{\lambda_{1}}{f_{1}}-\frac{\lambda_{2}}{f_{2}} & \mu^{2} \gamma_{n} \gamma_{u} \frac{\lambda_{1}}{f_{1}} \\
\operatorname{sym} & \lambda_{1}-\gamma_{u}^{2} \frac{\lambda_{1}}{f_{1}}
\end{array}\right]\right) \\
& =\left(-\mu^{2} \lambda_{1}-\mu^{4} \gamma_{n}^{2} \frac{\lambda_{1}}{f_{1}}-\frac{\lambda_{2}}{f_{2}}\right)\left(\lambda_{1}-\gamma_{u}^{2} \frac{\lambda_{1}}{f_{1}}\right)-\left(\mu^{2} \gamma_{n} \gamma_{u} \frac{\lambda_{1}}{f_{1}}\right)^{2} \\
& =-\mu^{2} \lambda_{1}^{2}-\mu^{4} \gamma_{n}^{2} \frac{\lambda_{1}^{2}}{f_{1}}-\frac{\lambda_{1} \lambda_{2}}{f_{2}}+\mu^{2} \gamma_{u}^{2} \frac{\lambda_{1}^{2}}{f_{1}}+\mu^{4} \gamma_{n}^{2} \gamma_{u}^{2} \frac{\lambda_{1}^{2}}{f_{1}^{2}}+\gamma_{u}^{2} \frac{\lambda_{1} \lambda_{2}}{f_{1} f_{2}}-\mu^{4} \gamma_{n}^{2} \gamma_{u}^{2} \frac{\lambda_{1}^{2}}{f_{1}^{2}} \\
& =-\mu^{2} \lambda_{1}^{2}-\mu^{4} \gamma_{n}^{2} \frac{\lambda_{1}^{2}}{f_{1}}-\frac{\lambda_{1} \lambda_{2}}{f_{2}}+\mu^{2} \gamma_{u}^{2} \frac{\lambda_{1}^{2}}{f_{1}}+\gamma_{u}^{2} \frac{\lambda_{1} \lambda_{2}}{f_{1} f_{2}},
\end{aligned}
$$

where $-\lambda_{1} \lambda_{2} / f_{2}>0$, and $\gamma_{u}^{2} \lambda_{1} \lambda_{2} /\left(f_{1} f_{2}\right)>0$. We also have

$$
\begin{aligned}
-\mu^{2} \lambda_{1}^{2}-\mu^{4} \gamma_{n}^{2} \frac{\lambda_{1}^{2}}{f_{1}}+\mu^{2} \gamma_{u}^{2} \frac{\lambda_{1}^{2}}{f_{1}} & =-\frac{\mu^{2} \lambda_{1}^{2}}{f_{1}}\left(f_{1}+\mu^{2} \gamma_{n}^{2}-\gamma_{u}^{2}\right) \\
& =-\frac{\mu^{2} \lambda_{1}^{2}}{f_{1}}\left(\frac{1}{2} \gamma_{u}^{2}+\frac{1}{2} \gamma_{v}^{2}-\frac{1}{2} \mu^{2} \gamma_{n}^{2}+\mu^{2} \gamma_{n}^{2}-\gamma_{u}^{2}\right) \\
& =-\frac{\mu^{2} \lambda_{1}^{2}}{f_{1}}\left(\frac{1}{2} \gamma_{v}^{2}-\frac{1}{2} \gamma_{u}^{2}+\frac{1}{2} \mu^{2} \gamma_{n}^{2}\right),
\end{aligned}
$$

$-\mu^{2} \lambda_{1}^{2} / f_{1}>0$, given $\gamma_{u}^{2}+\gamma_{v}^{2}<\mu^{2} \gamma_{n}^{2}$,

$$
\frac{1}{2} \gamma_{v}^{2}-\frac{1}{2} \gamma_{u}^{2}+\frac{1}{2} \mu^{2} \gamma_{n}^{2}>\frac{1}{2} \gamma_{v}^{2}-\frac{1}{2} \gamma_{u}^{2}+\frac{1}{2}\left(\gamma_{u}^{2}+\gamma_{v}^{2}\right)=\gamma_{v}^{2}>0,
$$


and therefore, $\Delta_{2}>0$. Finally,

$$
\begin{aligned}
\Delta_{3}= & \operatorname{det}\left(\left[\begin{array}{ccc}
-\mu^{2} \lambda_{1}-\mu^{4} \gamma_{n}^{2} \frac{\lambda_{1}}{f_{1}}-\frac{\lambda_{2}}{f_{2}} & \mu^{2} \gamma_{n} \frac{\gamma_{u} \frac{\lambda_{1}}{f_{1}}}{f_{1}} & \mu^{2} \gamma_{n} \gamma_{w} \frac{\lambda_{1}}{f_{1}} \\
\operatorname{sym} & \lambda_{1}-\gamma_{u}^{2} \frac{\lambda_{1}}{f_{1}} & -\gamma_{u} \gamma_{w} \frac{\lambda_{1}}{f_{1}} \\
= & \lambda_{1}-\gamma_{w}^{2} \frac{\lambda_{1}}{f_{1}}
\end{array}\right]\right) \\
& \left(-\mu^{2} \lambda_{1}-\mu^{4} \gamma_{n}^{2} \frac{\lambda_{1}}{f_{1}}-\frac{\lambda_{2}}{f_{2}}\right)\left|\begin{array}{cc}
\lambda_{1}-\gamma_{u}^{2} \frac{\lambda_{1}}{f_{1}} & -\gamma_{u} \gamma_{w} \frac{\lambda_{1}}{f_{1}} \\
-\gamma_{u} \gamma_{w} \frac{\lambda_{1}}{f_{1}} & \lambda_{1}-\gamma_{w}^{2} \frac{\lambda_{1}}{f_{1}}
\end{array}\right| \\
& +\left(\mu^{2} \gamma_{n} \gamma_{u} \frac{\lambda_{1}}{f_{1}}\right)\left|\begin{array}{ll}
\mu^{2} \gamma_{n} \gamma_{u} \frac{\lambda_{1}}{f_{1}} & -\gamma_{u} \gamma_{w} \frac{\lambda_{1}}{f_{1}} \\
\mu^{2} \gamma_{n} \gamma_{w} \frac{\lambda_{1}}{f_{1}} & \lambda_{1}-\gamma_{w}^{2} \frac{\lambda_{1}}{f_{1}}
\end{array}\right| \\
& +\left(\mu_{1} \frac{\lambda_{1}}{f_{1}}\right)\left|\begin{array}{ll}
\mu^{2} \gamma_{n} \gamma_{u} \frac{\lambda_{1}}{f_{1}} & \lambda_{1}-\gamma_{u}^{2} \frac{\lambda_{1}}{f_{1}} \\
\mu^{2} \gamma_{n} \gamma_{w} \frac{\lambda_{1}}{f_{1}} & -\gamma_{u} \gamma_{w} \frac{\lambda_{1}}{f_{1}}
\end{array}\right|,
\end{aligned}
$$

where,

$$
\begin{aligned}
\left|\begin{array}{ll}
\lambda_{1}-\gamma_{u}^{2} \frac{\lambda_{1}}{f_{1}} & -\gamma_{u} \gamma_{w} \frac{\lambda_{1}}{f_{1}} \\
-\gamma_{u} \gamma_{w} \frac{\lambda_{1}}{f_{1}} & \lambda_{1}-\gamma_{w}^{2} \frac{\lambda_{1}}{f_{1}}
\end{array}\right|=\lambda_{1}^{2}-\gamma_{u}^{2} \frac{\lambda_{1}^{2}}{f_{1}}-\gamma_{w}^{2} \frac{\lambda_{1}^{2}}{f_{1}} \\
\left|\begin{array}{cc}
\mu^{2} \gamma_{n} \gamma_{u} \frac{\lambda_{1}}{f_{1}} & -\gamma_{u} \gamma_{w} \frac{\lambda_{1}}{f_{1}} \\
\mu^{2} \gamma_{n} \gamma_{w} \frac{\lambda_{1}}{f_{1}} & \lambda_{1}-\gamma_{w}^{2} \frac{\lambda_{1}}{f_{1}}
\end{array}\right|=\mu^{2} \gamma_{n} \gamma_{u} \frac{\lambda_{1}^{2}}{f_{1}} \\
\left|\begin{array}{ll}
\mu^{2} \gamma_{n} \gamma_{u} \frac{\lambda_{1}}{f_{1}} & \lambda_{1}-\gamma_{u}^{2} \frac{\lambda_{1}}{f_{1}} \\
\mu^{2} \gamma_{n} \gamma_{w} \frac{\lambda_{1}}{f_{1}} & -\gamma_{u} \gamma_{w} \frac{\lambda_{1}}{f_{1}}
\end{array}\right|=-\mu^{2} \gamma_{n} \gamma_{w} \frac{\lambda_{1}^{2}}{f_{1}}
\end{aligned}
$$

therefore,

$$
\begin{aligned}
\Delta_{3} & =\left(-\mu^{2} \lambda_{1}-\mu^{4} \gamma_{n}^{2} \frac{\lambda_{1}}{f_{1}}-\frac{\lambda_{2}}{f_{2}}\right)\left(\lambda_{1}^{2}-\gamma_{u}^{2} \frac{\lambda_{1}^{2}}{f_{1}}-\gamma_{w}^{2} \frac{\lambda_{1}^{2}}{f_{1}}\right) \\
& -\left(\mu^{2} \gamma_{n} \gamma_{u} \frac{\lambda_{1}}{f_{1}}\right)\left(\mu^{2} \gamma_{n} \gamma_{u} \frac{\lambda_{1}^{2}}{f_{1}}\right)+\left(\mu^{2} \gamma_{n} \gamma_{w} \frac{\lambda_{1}}{f_{1}}\right)\left(-\mu^{2} \gamma_{n} \gamma_{w} \frac{\lambda_{1}^{2}}{f_{1}}\right)
\end{aligned}
$$

We have

$$
-\frac{\lambda_{2}}{f_{2}}\left(\lambda_{1}^{2}-\gamma_{u}^{2} \frac{\lambda_{1}^{2}}{f_{1}}-\gamma_{w}^{2} \frac{\lambda_{1}^{2}}{f_{1}}\right)>0,
$$

and the remaining parts

$$
\begin{aligned}
& \left(-\mu^{2} \lambda_{1}-\mu^{4} \gamma_{n}^{2} \frac{\lambda_{1}}{f_{1}}\right)\left(\lambda_{1}^{2}-\gamma_{u}^{2} \frac{\lambda_{1}^{2}}{f_{1}}-\gamma_{w}^{2} \frac{\lambda_{1}^{2}}{f_{1}}\right)-\mu^{4} \gamma_{n}^{2} \gamma_{u}^{2} \frac{\lambda_{1}^{3}}{f_{1}^{2}}-\mu^{4} \gamma_{n}^{2} \gamma_{w}^{2} \frac{\lambda_{1}^{3}}{f_{1}^{2}} \\
= & -\mu^{2} \lambda_{1}^{3}+\mu^{2} \gamma_{u}^{2} \frac{\lambda_{1}^{3}}{f_{1}}+\mu^{2} \gamma_{w}^{2} \frac{\lambda_{1}^{3}}{f_{1}}-\mu^{4} \gamma_{n}^{2} \frac{\lambda_{1}^{3}}{f_{1}} \\
= & -\mu^{2} \lambda_{1}^{3} / f_{1}\left(f_{1}-\gamma_{u}^{2}-\gamma_{w}^{2}+\mu^{2} \gamma_{n}^{2}\right),
\end{aligned}
$$


where $-\mu^{2} \lambda_{1}^{3} / f_{1}>0$, and

$$
\begin{aligned}
f_{1}-\gamma_{u}^{2}-\gamma_{w}^{2}+\mu^{2} \gamma_{n}^{2} & =\frac{1}{2}\left(\gamma_{u}^{2}+\gamma_{v}^{2}-\mu^{2} \gamma_{n}^{2}\right)-\gamma_{u}^{2}-\gamma_{w}^{2}+\mu^{2} \gamma_{n}^{2} \\
& =\frac{1}{2}\left(-\gamma_{u}^{2}-\gamma_{w}^{2}+\mu^{2} \gamma_{n}^{2}\right)>0 .
\end{aligned}
$$

Therefore, $\Delta_{3}>0$. Since all the principal minors of the $3 \times 3$ block matrix are positive, according to the Sylvester's criterion, the block matrix $\hat{\mathbf{D}}_{i}$ is positive definite. The block diagonal matrix $\hat{\mathbf{D}}$ assembled from the individual blocks is also positive-definite.

Corollary: The matrix $\mathbf{N}+\hat{\mathbf{M}}-\mathbf{B D}^{-1} \mathbf{C}$ is positive definite.

Proof: Since $\mathbf{N}$ is symmetric positive-semidefinite and $\hat{\mathbf{D}}$ is positive definite, it follows that $\mathbf{N}+\hat{\mathbf{D}}=\mathbf{N}+\hat{\mathbf{M}}-\mathbf{B D}^{-1} \mathbf{C}$ is positive definite. 